\title{
Dispepsia funcional y gastroparesia: ¿dos entidades superpuestas? De la fisiopatología a la terapéutica
}

\author{
María Mercedes Manresa, ${ }^{1}$ Paula Cecilia Carboné, ${ }^{2}$ Guillermina Diez ${ }^{3}$ \\ ${ }^{1}$ Sección Neurogastroenterología, Instituto de Gastroenterología Salim, Salta, Argentina. \\ ${ }^{2}$ Sección Neurogastroenterología, Instituto de Diagnóstico Médico Oroño, Rosario, Argentina. \\ ${ }^{3}$ Sección Neurogastroenterología, Centro Médico Olascoaga, Neuquén, Argentina.
}

Acta Gastroenterol Latinoam 2021;51(2):143-162

Recibido: 16/05/2021 / Aceptado: 26/05/2021 / Publicado online: 21/06/2021 / https://doi.org/10.52787/bvbw1724

\begin{abstract}
Resumen
La dispepsia funcional y la gastroparesia representan los trastornos sensitivo-motores más frecuentes del tracto gastrointestinal superior. Los avances cientificos de la última década han demostrado que existe una superposición significativa entre ambos trastornos. Esta revisión tiene como objetivo abordar los conocimientos actuales sobre fisiopatología, diagnóstico y tratamiento de dispepsia funcional y de gastroparesia. La dispepsia funcional es una condición médica que se caracteriza por uno o más de los siguientes sintomas: saciedad precoz, plenitud posprandial, dolor o ardor epigástrico. Los mecanismos fisiopatológicos involucrados son: las anormalidades de la motilidad y sensorialidad gastroduodenal, la disfunción inmune, la inflamación duodenal, las infecciones gastrointestinales, las alteraciones en la microbiota gastrointestinal y la disfunción del eje cerebro-intestino. Los criterios de Roma IV permiten establecer un diagnóstico presuntivo de dispepsia funcional en la mayoría de los pacientes, aunque
\end{abstract}

Correspondencia: María Mercedes Manresa

Correo electrónico: dramercedesmanresa@gmail.com continúa siendo un diagnóstico de exclusión que requiere la realización de una videoendoscopía digestiva alta en el caso de requerirse. Las opciones terapéuticas recomendadas son: la erradicación de Helicobacter pylori, los inhibidores de la bomba de protones, los antidepresivos tricíclicos y los proquinéticos. La gastroparesia es un trastorno crónico que se caracteriza por un retraso en el vaciamiento gástrico en ausencia de una obstrucción mecánica. Su fisiopatología se origina por anormalidades neuromusculares de la función motora gástrica. La gammagrafia para medición de vaciamiento gástrico es el gold standard actual para el diagnóstico. Los pilares del tratamiento son la restauración de la hidratación y la nutrición y el tratamiento farmacológico con proquinéticos $y$ antieméticos.

Palabras claves. Dispepsia funcional, gastroparesia, trastornos del tracto gastrointestinal superior, fisiopatología, diagnóstico, tratamiento.

\section{Functional Dyspepsia and Gastroparesis: Two Overlapping Entities? From Pathophysiology to Therapeutics}

\section{Summary}

Functional dyspepsia and gastroparesis represent the most common sensory-motor disorders of the upper gastrointestinal tract. Scientific advances in the last decade have shown that there is significant overlap between them. This review aims to address current knowledge about their pathophysiology, diagnosis and treatment. Functional dyspepsia is a medical condition that is characterized by one or more of the 
following symptoms: early satiety, postprandial fullness, and epigastric pain or burning. The pathophysiological mechanisms involved are: the gastroduodenal motility and sensory abnormalities, the immune dysfunction, the duodenal inflammation, the gastrointestinal infections, the alterations in the gastrointestinal microbiota and the dysfunction of the gut-brain axis. The Rome IV criteria make possible to establish a presumptive diagnosis of functional dyspepsia in most patients, although it continues to be a diagnosis of exclusion that requires the performance of an upper digestive video endoscopy to confirm it. The recommended therapeutic options are: the eradication of Helicobacter pylori, the proton pump inhibitors, the tricyclic antidepressants and prokinetics. Gastroparesis is a chronic disorder characterized by delayed gastric emptying in the absence of a mechanical obstruction. The pathophysiology is caused by neuromuscular abnormalities of gastric motor function. Gastric emptying scan is the current gold standard for diagnosis. The mainstays of treatment are restoration of hydration and nutrition, and pharmacological treatment with prokinetics and antiemetics.

Keywords. Functional dyspepsia, gastroparesis, upper gastrointestinal disorders, pathophysiology, diagnosis, treatment.

\section{Abreviaturas}

DF: Dispepsia funcional.

Gp: Gastroparesia.

VGR: Vaciamiento gástrico retrasado.

SDP: Sindrome de distrés posprandial.

SDE: Sindrome de dolor epigástrico.

VEDA: Videoendoscopia digestiva alta.

H. pylori: Helicobacter pylori.

SII: Sindrome del intestino irritable.

ERGE: Enfermedad por reflujo gastroesofágico.

$V G$ : Vaciamiento gástrico.

VGR: Vaciamiento gástrico retrasado.

DF-PI: Dispepsia funcional posinfecciosa.

SIBO: Sobrecrecimiento bacteriano del intestino delgado.

SNC: Sistema nervioso central.

RMN: Resonancia magnética nuclear.

ACG: Sigla en inglés de Colegio Americano de Gastroenterología.

CAG: Sigla en inglés de Asociación Canadiense de Gastroenterología.

NNT: Número necesario a tratar.

IBP: Inhibidores de la bomba de protones.
FDA: Sigla en inglés de administración de alimentos y medicamentos.

ATC: Antidepresivos tricíclicos.

ECA: Ensayo controlado aleatorizado.

ISRS: Inhibidores selectivos de la recaptación de serotonina.

DM: Diabetes mellitus.

GVG: Gammagrafía de vaciamiento gástrico.

5-HT4: 5-hidroxitriptamina tipo 4.

EEG: Estimulación eléctrica gástrica.

G-POEM: Sigla en inglés de miotomía endoscópica peroral gástrica.

\section{Introducción}

La dispepsia funcional (DF) y la gastroparesia $(\mathrm{Gp})$ representan los trastornos sensitivo-motores más frecuentes del tracto gastrointestinal superior. ${ }^{1}$ Históricamente, se los ha considerado como dos trastornos gastrointestinales completamente diferentes. Sin embargo, los avances científicos de la última década han demostrado que existe una superposición significativa entre ambos. ${ }^{2}$ Los síntomas referidos por los pacientes no pueden predecir de manera confiable la fisiopatología subyacente, lo que impide la discriminación entre la DF y la Gp a través de la clínica. ${ }^{3-7}$

Actualmente, se considera que la DF y la Gp forman parte de un espectro amplio y continuo de disfunción sensitivo-motora gastroduodenal, con similitudes y diferencias entre ambas. ${ }^{1}$

Esta revisión tiene como objetivo abordar los conocimientos actuales sobre fisiopatología, diagnóstico y tratamiento de la DF y la Gp.

\section{Dispepsia funcional}

La DF es una condición médica que impacta de forma significativa en las actividades habituales de un paciente y se caracteriza por uno o más de los siguientes síntomas: saciedad precoz, plenitud posprandial, dolor o ardor epigástrico, los cuales no se pueden explicar luego de una evaluación clínica de rutina. ${ }^{8}$ Se clasifica en síndrome de distrés posprandial (SDP), el cual se caracteriza por síntomas inducidos por las comidas, síndrome de dolor epigástrico (SDE), que puede ocurrir en el periodo posprandial, durante el ayuno, e incluso puede mejorar con la ingesta de alimentos y el síndrome de superposición entre ambos síndromes. Pueden estar presentes otros síntomas como la sensación de hinchazón posprandial a nivel epigástrico, los eructos excesivos y las náuseas, aunque estos no formen parte de los criterios diagnósticos. Los vómitos no son frecuentes y si persisten es necesario descartar la presencia de causas orgánicas y de Gp.? 
Aproximadamente el $10 \%$ de la población cumple con los criterios de Roma IV para la DF, siendo el SDP (61\%) más prevalente que el SDE (18\%) y que la superposición entre ambos $(21 \%) .^{10}$

Factores como el sexo femenino, el tabaquismo, el uso de drogas antiinflamatorias no esteroideas y la infección por Helicobacter pylori (H pylori) se asociaron con la DF en la población general. ${ }^{11}$ Con respecto a la infección por H pylori, el consenso de Kioto sugiere que representa una causa orgánica de dispepsia. ${ }^{12}$

La DF se asocia con los trastornos psicológicos, ${ }^{13-15}$ la somatización, ${ }^{14,16}$ el deterioro de la calidad de vida ${ }^{17}$ y con otros trastornos gastrointestinales funcionales como el síndrome del intestino irritable (SII) y la enfermedad por reflujo gastroesofágico (ERGE). ${ }^{18,19}$

\section{Fisiopatología}

Como consecuencia de su naturaleza compleja y de la heterogeneidad de sus síntomas, los mecanismos fisiopatológicos subyacentes de la DF permanecen aún poco claros.

Considerado anteriormente como un desorden originado de forma exclusiva en alteraciones gástricas motoras y sensoriales, en la actualidad hay fuerte evidencia sobre que el duodeno es clave en la patogenia de la DF. ${ }^{20}$

Varios mecanismos fisiopatológicos parecen estar involucrados en el desarrollo de los síntomas de la DF.

\section{Disfunción motora y sensorial gastroduodenal}

\section{- Alteraciones de la motilidad gastroduodenal}

Tradicionalmente, la DF ha sido atribuida a factores fisiológicos gástricos como el retraso del vaciamiento gástrico (VG), la falla en la acomodación gástrica posterior a una comida o la hipersensibilidad gástrica frente a la distensión del estómago. ${ }^{21}$ No obstante, no se logró establecer una correlación entre las alteraciones motoras gástricas con ninguno de los subtipos de la DF (SDP/SDE). ${ }^{22}$

Las alteraciones del VG pueden ser identificadas en aproximadamente $1 / 3$ de los pacientes con DF, pudiendo encontrarse tanto vaciamiento retrasado como acelerado. ${ }^{23,24} \mathrm{El}$ vaciamiento gástrico retrasado (VGR) se reportó en cerca del $30 \%$ de los pacientes con DF. ${ }^{22,25} \mathrm{Se}$ ha evidenciado una inconsistencia entre la tasa de VG y los síntomas. ${ }^{26,27}$ También, son variables los resultados de los estudios que correlacionan la mejoría sintomática con la aceleración en el VG. ${ }^{28,29}$

En un $40 \%$ de los pacientes con DF, puede identificarse un deterioro en la acomodación gástrica, ${ }^{30}$ que resulta en la distribución de mayores volúmenes a nivel antral y menores en el fundus..$^{31,32}$ El grado de distensión antral se ha relacionado con la severidad de los síntomas de la DF. ${ }^{33}$ La alteración de la acomodación gástrica se asoció con la saciedad precoz. ${ }^{27}$

\section{- Hipersensibilidad visceral gastroduodenal}

Los pacientes con DF pueden presentar hipersensibilidad visceral frente a la distensión gástrica ${ }^{22,34} \mathrm{y}$, en algunos casos, a la distensión duodenal, ${ }^{35}$ yeyunal ${ }^{36} \mathrm{o}$ rectal. ${ }^{37} \mathrm{Su}$ grado se correlaciona con la severidad de los síntomas. ${ }^{38}$ Se ha demostrado que la hipersensibilidad a la distensión gástrica se asocia con el dolor epigástrico y con sensaciones no dolorosas como la plenitud posprandial, la hinchazón abdominal y los eructos. ${ }^{39,40}$

Se evidenciaron una sensibilidad aumentada al ácido en el duodeno y un aclaramiento disminuido del ácido desde el bulbo duodenal, los cuales se relacionaron con las náuseas. ${ }^{41,42} \mathrm{~A}$ su vez, se demostró que la administración exógena de ácido en el duodeno disminuye el umbral de sensibilidad frente a la distensión gástrica con balón e inhibe la acomodación gástrica posprandial. ${ }^{43,44}$

La infusión intraduodenal de lípidos sensibiliza al estómago a la distensión en pacientes con $\mathrm{DF},{ }^{45}$ lo que podría explicar el motivo por el cual las comidas grasas pueden empeorar los síntomas relacionados al llenado gástrico.

\section{Disfunción inmune - inflamación duodenal}

Cada vez es mayor la evidencia sobre la presencia de una inflamación de bajo grado en la mucosa duodenal en los pacientes con DF, incluyendo un número aumentado de eosinófilos, mastocitos y células T. ${ }^{46,47}$

La inflamación duodenal se ha reportado en hasta un $40 \%$ de los casos de DF, principalmente eosinofilia duodenal con degranulación adyacente a los nervios. ${ }^{48,49}$ El primer reporte en adultos data del año 2007, cuando Talley y col. ${ }^{48}$ demostraron que un recuento más alto de eosinófilos en la mucosa de la primera y segunda porción duodenal se asoció con una mayor probabilidad de diagnóstico de DF, concluyendo que la eosinofilia duodenal puede caracterizar a un subgrupo de los pacientes con dispepsia, particularmente los pacientes con el SDP. ${ }^{50}$ Hallazgos similares se replicaron en nuevos estudios alrededor del mundo. ${ }^{47,49,51,53,58}$

En un estudio reciente, la eosinofilia duodenal se asoció con un riesgo aumentado de ERGE a los 10 años de seguimiento en los pacientes con DF de base, lo cual sugiere que la eosinofilia duodenal podría explicar, en parte, la relación entre la ERGE y la DF.52

Con respecto al recuento y la tasa de degranulación de 
mastocitos, los resultados son variables; ${ }^{53,54}$ sin embargo, se observó una diferencia significativa en su contenido granular. ${ }^{55}$

La liberación de mediadores inflamatorios, por parte de los eosinófilos y los mastocitos, induce daño tisular y potencial disfunción de la barrera epitelial. El aumento de la permeabilidad epitelial puede permitir la infiltración de antígenos luminales secundarios, perpetuando la respuesta inmune y favoreciendo la aparición de los síntomas. ${ }^{56}$

En distintos estudios, se ha podido evidenciar la alteración de la función de barrera del epitelio duodenal, demostrando una expresión reducida de proteínas de adhesión de célula a célula, ${ }^{46}$ así como valores disminuidos de impedancia basal en los pacientes con DF. ${ }^{57}$

Cirillo y col. demostraron un número aumentado de eosinófilos y mastocitos en la proximidad de los ganglios del plexo entérico submucoso, corroborando alteraciones funcionales y estructurales a este nivel. ${ }^{58}$

Otra evidencia que relaciona la activación inmune anormal con la DF es el hallazgo de un aumento de linfocitos $T$ integrina $\alpha 4 \beta 7+$ /receptor 9 de quimiocina+, el cual se asoció con niveles elevados de citoquinas en la circulación periférica, con una mayor intensidad de los síntomas y con el VGR. ${ }^{59}$

\section{Infecciones gastrointestinales}

Las infecciones gastrointestinales agudas pueden desencadenar la DF en un 10 a un $20 \%$ de los pacientes. ${ }^{60-62}$ Mearin y col. reportaron que, luego de una gastroenteritis aguda por Salmonella enteritidis, el riesgo de padecer dispepsia funcional posinfecciosa (DF-PI) es 5 veces mayor que en los sujetos no expuestos. ${ }^{63}$ Otros patógenos que se han asociado con la DF-PI son Escherichia coli O157, Campylobacter jejuni, Giardia lamblia y Norovirus. ${ }^{62,64,65}$

Los factores de riesgo relacionados con la DF-PI fueron el distrés psicológico, los factores genéticos y el tabaquismo. ${ }^{66,67}$

La DF-PI se asoció con la presencia de infiltrado inflamatorio duodenal con eosinófilos y macrófagos CCR2 $+{ }^{49}$ También, se encontró un deterioro de la acomodación gástrica que se atribuyó a una disfunción a nivel de las neuronas gástricas nitrinérgicas. ${ }^{60}$

\section{Alteración de la microbiota gastrointestinal}

Mientras el rol de la disbiosis intestinal y el sobrecrecimiento bacteriano del intestino delgado (SIBO, por su sigla en inglés) han sido focos de gran interés en otros trastornos funcionales gastrointestinales como el SII, ${ }^{68}$ es relativamente poco lo que se conoce sobre la microbiota y su relación potencial con la $\mathrm{DF}$.
En un estudio reciente, se evidenció una disminución significativa de los géneros anaeróbicos como Prevotella, Veillonella y Actinomyces en los pacientes con DF. Se observó que la carga bacteriana de la mucosa duodenal se correlacionó con síntomas más severos en respuesta a una comida $(p=0,02){ }^{69}$

También, se han encontrado alteraciones en la microbiota del fluido gástrico en los pacientes con DF, siendo más abundantes los Bacteroidetes con menor cantidad de Proteobacteria y ausencia de Acidobacteria. Se sugirió al reflujo del contenido del intestino delgado al estómago como responsable de este cambio en la composición bacteriana. $^{70}$

Es contradictoria la evidencia sobre el rol del SIBO en la DF. ${ }^{71,72}$

\section{Disfunción del eje cerebro-intestino}

El distrés psicológico, particularmente la ansiedad, se asocia con la DF. En un seguimiento a 10 años, la ansiedad de base, no así la depresión, aumentó el riesgo de desarrollar DF en casi 8 veces. La ansiedad se asoció particularmente con el SDP, pero no con el SDE. ${ }^{73}$ De manera contraria, se evidenció que, entre los pacientes con desórdenes funcionales gastrointestinales de base, sin niveles elevados de ansiedad y depresión, hubo significativamente mayores niveles de ansiedad y depresión en el seguimiento. Esto sugiere que el sistema nervioso central (SNC) y el intestino interactúan bidireccionalmente en los desórdenes funcionales gastrointestinales. ${ }^{74,75}$

Se ha observado un procesamiento central anormal del dolor en los pacientes con DF. ${ }^{76}$ Los estudios con resonancia magnética nuclear (RMN) funcional de los pacientes con DF demostraron anormalidades de la conectividad funcional y estructural en las áreas cerebrales responsables de procesar la información visceral aferente..$^{77,78}$ Estas alteraciones fueron más marcadas en el SDE que en el SDP.

Los desórdenes del eje cerebro-intestino pueden inducir una disfunción de la barrera epitelial intestinal. Hay una fuerte evidencia que indica que el estrés, a través de este eje, puede causar la disfunción de la barrera epitelial intestinal, principalmente vía liberación sistémica y periférica del factor liberador de corticotropina. ${ }^{79,80}$

\section{Diagnóstico}

La primera etapa del abordaje diagnóstico consiste en realizar una cuidadosa historia clínica, interrogando sobre los síntomas incluidos en los criterios de Roma IV (Tabla 1). Esto nos permitirá clasificar a los pacientes en el subtipo apropiado, ${ }^{9}$ teniendo en cuenta que pueden 


\section{Tabla 1. Criterios de Roma IV}

B1. Dispepsia funcional

Criterios de diagnóstico*

1. Uno o más de los siguientes:
a. Plenitud posprandial molesta.
b. Saciedad precoz molesta.
c. Dolor epigástrico molesto.
d. Ardor epigástrico molesto.

2. Sin evidencia de enfermedad estructural (incluyendo una VEDA) que permita explicar los síntomas.

* Deben cumplirse los criterios de B1a y/o B1b.

* Los criterios deben estar presentes durante los últimos tres meses, con inicio de los síntomas al menos seis meses antes del diagnóstico.

B1a. Síndrome de distrés posprandial (SDP)

Criterios de diagnóstico*

Debe incluir uno 0 ambos de los siguientes, al menos tres días por semana:

1. Plenitud posprandial molesta (es decir, que produce un impacto en las actividades habituales).

2. Saciedad precoz molesta (es decir, que impide terminar una comida de tamaño regular).

Sin evidencia de enfermedad orgánica, sistémica o metabólica que permita explicar los síntomas en las investigaciones de rutina (incluyendo una VEDA).

* Los criterios deben cumplirse durante los últimos tres meses, con inicio de los síntomas al menos seis meses antes del diagnóstico.

Comentarios de apoyo:

- También pueden estar presentes: dolor o ardor epigástrico posprandial, hinchazón en el epigastrio, eructos excesivos y náuseas.

- La presencia de vómitos justifica la consideración de otro trastorno.

- La pirosis no es un síntoma de dispepsia, pero puede coexistir con frecuencia.

- Los síntomas que alivian con la evacuación de heces o gases generalmente no deben considerarse parte de la dispepsia.

B1b. Síndrome de dolor epigástrico (SDE)

Criterios de diagnóstico*

Debe incluir al menos uno de los siguientes síntomas, al menos un día a la semana:

1. Dolor epigástrico molesto (es decir, que produce un impacto en las actividades habituales).

2. Ardor epigástrico molesto (es decir, que produce un impacto en las actividades habituales).

Sin evidencia de enfermedad orgánica, sistémica o metabólica que permita explicar los síntomas en las investigaciones de rutina (incluyendo una VEDA).

* Los criterios deben cumplirse durante los últimos tres meses, con inicio de los síntomas al menos seis meses antes del diagnóstico.

Comentarios de apoyo:

- El dolor puede ser inducido por la ingesta de una comida, aliviado con la ingesta de una comida, o puede ocurrir durante el ayuno.

- También pueden estar presentes: hinchazón epigástrica posprandial, eructos y náuseas.

- Los vómitos persistentes probablemente sugieren otro trastorno.

- La pirosis no es un síntoma de dispepsia, pero puede coexistir con frecuencia.

- El dolor no debe cumplir con los criterios de dolor biliar.

- Los síntomas que alivian con la evacuación de heces o gases generalmente no deben considerarse parte de la dispepsia. 
presentar superposición entre los mismos. Resulta relevante investigar sobre la presencia de síntomas o signos de alarma que podrían sugerir un diagnóstico alternativo (por ejemplo, pérdida de peso, anemia, disfagia o vómitos persistentes). La siguiente etapa incluye el examen físico que, aunque generalmente resulta normal, le brinda tranquilidad al paciente. ${ }^{81}$

La historia clínica y el examen físico no pueden distinguir con precisión entre la DF y la orgánica. Desafortunadamente, no se dispone de un biomarcador y no existe un algoritmo diagnóstico validado. No se recomienda realizar pruebas de laboratorio de rutina en todos los pacientes, ${ }^{9,} 82$ aunque puede ser de importancia solicitar un hemograma para descartar la presencia de anemia. Se justifica realizar pruebas de laboratorio adicionales ante la sospecha de una posible causa biliar. La ecografía o la tomografía computada de abdomen no se recomiendan de rutina en ausencia de síntomas o signos de alarma o sugestivos de patología biliopancreática, ya que el rendimiento diagnóstico es bajo. ${ }^{83}$

La mayoría de los pacientes con dispepsia (82\%) no muestra hallazgos endoscópicos patológicos: alrededor del $10 \%$ presenta una úlcera péptica y menos del $0,5 \%$, cáncer gastroesofágico. ${ }^{84} \mathrm{Si}$ bien se requiere de una VEDA negativa para confirmar el diagnóstico de DF, 'la necesidad de realizarla se tiene que evaluar en cada paciente en particular. La decisión depende fundamentalmente de los factores de riesgo para cáncer gastrointestinal alto como el sexo masculino, la edad, los antecedentes familiares de cáncer de esófago o estómago y el nacimiento o infancia en países con una elevada incidencia de cáncer gástrico. También, es importante tener en cuenta la presencia de síntomas o signos de alarma y la falta de respuesta al tratamiento. En la reciente guía conjunta del Colegio Americano de Gastroenterología y de la Asociación Canadiense de Gastroenterología (ACG y CAG, por sus siglas en inglés), se sugiere solicitar una VEDA a los pacientes con síntomas de dispepsia a partir de los 60 años. Esta recomendación es condicional y se basa en la incidencia del cáncer gástrico en Canadá y Estados Unidos y en análisis económicos realizados en Estados Unidos. ${ }^{82}$ Debido a la mayor incidencia de cáncer gástrico que hay en América del Sur, la cual es mayor en los países que limitan con el pacífico, la guía latinoamericana de dispepsia recomienda realizar una VEDA en los pacientes con inicio de los síntomas a los 40-45 años. ${ }^{85}$ No se recomienda la toma de biopsias duodenales si no existen otros síntomas o signos que incrementen el riesgo de detectar una enfermedad celíaca. ${ }^{86}$

En los pacientes que no tienen indicación de una VEDA, se recomienda realizar una prueba no invasiva para detectar $H$. pylori, como el antígeno fecal o la prueba del aliento con urea. ${ }^{82}$

En resumen, los criterios de Roma IV permiten establecer un diagnóstico presuntivo de DF en la mayoría de los pacientes sin síntomas o signos de alarma y sin factores de riesgo para enfermedades orgánicas, aunque continúa siendo un diagnóstico de exclusión que requiere la realización de una VEDA para confirmarlo en algunas situaciones. ${ }^{87}$

\section{Tratamiento}

El manejo de la DF incluye darle tranquilidad al paciente de que no existe una causa estructural para sus síntomas, explicarle la fisiopatogenia y la historia natural del trastorno y brindarle información sobre las limitaciones de las terapias disponibles, con el fin de manejar sus expectativas. ${ }^{88}$

Actualmente, existe poca evidencia para sugerir que un cambio en la dieta lleve a una mejoría de los síntomas de la DF. Si bien algunos alimentos se asociaron con la generación de síntomas de dispepsia, son escasos los ensayos clínicos que evalúan formalmente las intervenciones dietéticas para el tratamiento de la DF. ${ }^{89}$

La terapia médica es el pilar del tratamiento, aunque la mayoría de los fármacos tienen una eficacia limitada y ninguno ha demostrado alterar la historia natural a largo plazo de la DF. ${ }^{88}$ De forma reciente, la guía clínica del ACG y la CAG emitió recomendaciones, con respecto al tratamiento de la DF, en base a la evidencia disponible. ${ }^{82}$

\section{Erradicación de Helicobacter pylori}

La guía del ACG y la CAG recomienda erradicar $H$. pylori en todos los pacientes con DF que sean positivos para dicha bacteria. Con una calidad de evidencia alta, se ha estimado un número necesario a tratar (NNT) de 12,5 (IC 95\% 10-20). ${ }^{82}$ A pesar de que el impacto de la erradicación en los síntomas de la DF es leve (RR dispepsia persistente 0,91 ; IC 95\% 0,88-0,94; $p=0,00001$ ), la recomendación es fuerte debido a que el abordaje es costo efectivo ${ }^{90}$ y a que los eventos adversos asociados al uso de antibióticos generalmente son leves. A su vez, la erradicación de $H$. pylori puede reducir el riesgo futuro de cáncer gástrico y de enfermedad úlcero-péptica por lo que los beneficios de este enfoque superan claramente los daños.

La ganancia con respecto al control de los síntomas puede tardar al menos 6 meses en volverse significativa y esto se ha atribuido al tiempo que demora en resolverse la gastritis asociada a la infección. ${ }^{91-93}$

Es posible que la eficacia de este abordaje se relacione también con el tratamiento de otros agentes infecciosos que podrían causar la dispepsia. ${ }^{94}$ 


\section{Inhibidores de la bomba de protones}

La guía del ACG y la CAG recomienda tratar con inhibidores de la bomba de protones (IBP) a los pacientes con DF en ausencia de $H$. pylori y a los que permanecen sintomáticos a pesar de la erradicación de la infección. El tratamiento con IBP tiene un impacto estadísticamente significativo en los síntomas de la dispepsia (RR dispepsia persistente 0,87 ; IC 95\% 0,82-0,94; $p<0,00001$ ) con un NNT de 10 (IC 95\% 7-20). La recomendación es fuerte debido a que los IBP son bien tolerados y accesibles. La calidad de la evidencia disponible es moderada. No se observó un beneficio adicional al duplicar la dosis de los IBP por lo que la droga se debe discontinuar, si el paciente no responde luego de 8 semanas de la dosis estándar. No hay evidencia de que el SDE responda mejor a los IBP que el SDP por lo que, actualmente, no se recomienda utilizar el subtipo de la DF para guiar la elección del tratamiento. ${ }^{82}$ En cuanto a los riesgos a largo plazo del consumo del IBP, en la mayoría de las asociaciones descritas, el número necesario para dañar fue mayor a $1000,{ }^{95}$ por lo que los beneficios superan a los riesgos. A pesar de esto, la administración de alimentos y medicamentos (FDA, por su sigla en inglés) de Estados Unidos sugiere no continuar un tratamiento a largo plazo con los IBP sin un intento de suspenderlos cada 6-12 meses.

Al comparar los IBP con los proquinéticos, se observó una pequeña diferencia a favor de los primeros (RR dispepsia persistente 0,90 ; IC $95 \% 0,81-1 ; p=0,04)$. Sin embargo, la mayoría de los estudios involucrados en dicho análisis tienen problemas metodológicos que pueden influenciar en los resultados. ${ }^{96}$

En una investigación reciente, se mostró que los IBP mejoraron la inflamación de la mucosa duodenal y su disfunción de barrera. A su vez, los cambios en los eosinófilos duodenales se asociaron con la eficacia clínica de los IBP en la DF. ${ }^{97}$

\section{Neuromoduladores}

La guía del ACG y la CAG recomienda considerar el tratamiento con antidepresivos tricíclicos (ATC) en los pacientes con DF que no responden a la erradicación de $H$. pylori o a los IBP. ${ }^{82}$ Los ATC mostraron un efecto estadísticamente significativo en la reducción de los síntomas de dispepsia (RR 0,74; IC 95\% 0,61-0,91) con un NNT de 6 (IC 95\% 6-18). ${ }^{98}$ La recomendación es condicional debido a que los ATC se asocian con eventos adversos (constipación, boca seca, retención urinaria y somnolencia) y a que una proporción significativa de los pacientes prefieren no tomar medicamentos antidepresivos. La calidad de la evidencia disponible es moderada. ${ }^{82}$
En dos ensayos controlados aleatorizados (ECA), se compararon a los inhibidores selectivos de la recaptación de serotonina (ISRS) con placebo en los pacientes con DF sin encontrar un efecto estadísticamente relevante en los síntomas (RR 1,01; IC 95\% 0,89-1,15). ${ }^{99,100}$

La mirtazapina, un antidepresivo con actividad adrenérgica y serotoninérgica central, tiene potencial en el tratamiento de los pacientes con DF y con pérdida de peso. En un estudio piloto que evaluó a los pacientes con DF y con pérdida de peso, en ausencia de depresión o de un trastorno de ansiedad, dicho fármaco mejoró la saciedad precoz produciendo un aumento significativo de peso en comparación con el placebo. ${ }^{101}$ En un ECA realizado en pacientes con DF y depresión con pérdida de peso, se mostró una mayor eficacia que con paroxetina y que con el tratamiento convencional para aliviar los síntomas, produciendo un aumento del apetito y una ganancia significativa de peso. ${ }^{102}$

\section{Proquinéticos}

La guía del ACG y la CAG recomienda ofrecer tratamiento con proquinéticos a los pacientes con DF que no responden a la erradicación de $\mathrm{H}$. pylori, a los IBP o a los ATC. Dicha recomendación es condicional, con una calidad de evidencia muy baja. ${ }^{82}$ Los agentes proquinéticos fueron más efectivos que el placebo (RR 0,81; IC 95\% 0,74-0,89) con un NNT de 7 (IC 95\% 5-12). En la mayoría de los estudios evaluados, se utilizó cisaprida, la cual se asoció con la prolongación del intervalo QT y arritmias potencialmente mortales, por lo que se retiró del mercado en la mayoría de los países. Al eliminar dicho fármaco del análisis, los proquinéticos siguen siendo más efectivos que el placebo (RR 0,87; IC 95\% 0,80-0,94) con un aumento del NNT a 12 (IC 95\% 8-27). ${ }^{103}$ La evidencia sugiere un beneficio de los proquinéticos en el tratamiento de ambos subtipos de la DF por lo que, como se mencionó anteriormente, no se recomienda utilizar la clasificación de la DF para guiar la elección del tratamiento. Aparte de la cisaprida, los proquinéticos analizados parecen ser bien tolerados en los tratamientos a corto plazo. ${ }^{82}$

En el análisis por subgrupos, la acotiamida (RR 0,94; IC 95\% 0,91-0,98; NNT 20; IC 95\% 13-60) y el tegaserod (RR 0,89; IC 95\% 0,82-0,96; NNT 14, IC 95\% 8-38) fueron eficaces, en forma individual, en comparación con el placebo, mientras que con la itoprida se observó una tendencia al beneficio (RR 0,70; IC 95\% $0,47-1,03)$. No hay evidencia suficiente para concluir si algún proquinético es más efectivo que otro. La acotiamida, un inhibidor de acetilcolinesterasa que relaja el fundus gástrico, cuenta con licencia para su uso en pacientes con DF en algunos países como Japón e India. ${ }^{104}$ El tegaserod 
fue retirado del mercado, en la mayoría de los países, debido a sus posibles efectos adversos cardiovasculares. Sin embargo, en Estados Unidos, se reintrodujo en 2019 para su uso en mujeres con SII subtipo constipación con bajo riesgo cardiovascular. ${ }^{105,106}$

La domperidona fue evaluada en 7 ensayos clíni$\cos ^{107-113}$ que fueron excluidos del análisis realizado por el ACG y la CAG porque no cumplían con los criterios de elegibilidad. La guía expone los resultados de dichos estudios (RR 0,71; IC 95\% 0,53-0,97; NNT 3; IC 95\% 2-8) porque la domperidona es un fármaco disponible en muchos países, pero aclara que estos hallazgos deben ser interpretados con precaución. A su vez, es importante tener en cuenta que la domperidona puede prolongar el intervalo QT, aumentando el riesgo de arritmias graves en los pacientes con condiciones cardíacas preexistentes. ${ }^{82}$

En conclusión, los agentes proquinéticos son una opción terapéutica razonable para los pacientes con $\mathrm{DF}$ que no han respondido a otros fármacos. ${ }^{103}$

\section{Probióticos}

Como se mencionó anteriormente, de forma reciente se demostró que la inflamación de bajo grado y el aumento de la permeabilidad de la mucosa duodenal son mecanismos involucrados en la fisiopatogenia de la DF, ${ }^{46}$ por lo que se piensa que la modulación de la microbiota intestinal podría ser una opción terapéutica beneficiosa. En una revisión sistemática y un metanálisis reciente, ${ }^{114}$ se evaluaron 4 ECA que utilizaron diferentes especies de Lactobacillus, Bifidobacterium, Streptococcus o Bacillus ${ }^{15-118}$ en pacientes con DF sin demostrar un efecto en la mejoría de los síntomas.

\section{Rifaximina}

La rifaximina tiene múltiples mecanismos por los que podría conducir a una mejoría en los síntomas de DF. Se postula que previene la inflamación intestinal, reduce la hiperalgesia visceral y actúa como un modulador de la microbiota intestinal. ${ }^{119}$ Recientemente, se llevó a cabo un ECA que evaluó su efecto en los pacientes con DF sin SII. El fármaco fue superior al placebo en proporcionar una mejoría de los síntomas globales de dispepsia (78\% versus 52\%; OR 3,16; IC 95\% 1,23$8,09 ; p=0,02)$ y del síntoma plenitud postprandial en forma individual ( $80 \%$ versus $59 \%$; OR 2,82; IC $95 \%$ 1,07-7,44; $p=0,03) .{ }^{120}$ Se necesitan estudios de mayor tamaño para reproducir este hallazgo y establecer el mecanismo de acción de la rifaximina en esta posible indicación.

\section{Terapias psicológicas}

La guía del ACG y la CAG sugiere ofrecer terapias psicológicas a los pacientes con DF que no respondan al tratamiento con fármacos. Esta recomendación es condicional debido a que la calidad de la evidencia disponible es muy baja y a que requiere tiempo y motivación por parte del paciente. ${ }^{82}$ Los estudios analizados ${ }^{121-124}$ mostraron un beneficio significativo de las terapias psicológicas (RR 0,53; IC 95\% 0,44-0,65) con un NNT de 3 (IC 95\% 3-4). El abordaje más frecuente fue la terapia cognitivo-conductual. A pesar del efecto dramático en la reducción de los síntomas de dispepsia, la calidad de la evidencia es muy baja por lo que los hallazgos se deben interpretar con precaución.

\section{Gastroparesia}

La Gp es un trastorno crónico que se caracteriza por un retraso en el VG, después de una comida, en ausencia de una obstrucción mecánica. Los síntomas de presentación incluyen la saciedad precoz, la plenitud postprandial, las náuseas, los vómitos, el dolor epigástrico, la hinchazón y la distensión abdominal. ${ }^{125,126}$

Se estima que la prevalencia de la Gp, en la comunidad, es de 24,2 por 100.000 habitantes $(0,02 \%)$, siendo 4 veces mayor en mujeres que en hombres. ${ }^{127} \mathrm{~A}$ su vez, se calcula que el $1,8 \%$ de la población general podría tener un VGR por lo que la proporción de los pacientes con Gp diagnosticada reflejaría solo la punta del "iceberg". ${ }^{128}$

En la mayoría de las series, la Gp idiopática representa la categoría más frecuente. ${ }^{129}$ Otras causas habituales son la DM y la forma iatrogénica relacionada con fármacos o cirugías. ${ }^{130} \mathrm{La}$ Gp puede ocurrir luego de un proceso infeccioso viral ${ }^{131}$ o bacteriano. ${ }^{63}$ Puede relacionarse también con desórdenes neurológicos como la enfermedad de Parkinson y con colagenopatías como la esclerodermia. ${ }^{130}$ Otras causas menos usuales incluyen la disfunción tiroidea, la enfermedad crítica, la pseudoobstrucción intestinal crónica, la amiloidosis, la insuficiencia renal, la enfermedad paraneoplásica, la isquemia mesentérica, la enfermedad degenerativa mitocondrial y las miopatías viscerales, entre otras. ${ }^{126,129,130}$

\section{Fisiopatología}

La Gp representa un trastorno complejo que se origina por alteraciones neuromusculares de la función motora gástrica, ${ }^{132}$ con diversos mecanismos fisiopatológicos involucrados a nivel de la inervación entérica intrínseca, vagal extrínseca o muscular.

\section{Neuropatía intrínseca}

Se evidenció una disminución en el número de las neu- 
ronas inhibitorias nitrinérgicas en el $40 \%$ de los pacientes con Gp idiopática y en el 20\% de los pacientes con Gp diabética, con una consecuente alteración en la coordinación de la peristalsis gástrica y en la relajación pilórica. ${ }^{133}$

Se reportó también una disminución en el número de las células intersticiales de Cajal en el cuerpo gástrico de los pacientes con Gp diabética e idiopática; lo cual lleva a un deterioro en la conducción de la actividad eléctrica, interfiriendo con la peristalsis, la trituración y el VG. ${ }^{134,135}$

Se ha descrito que el daño sobre las células intersticiales de Cajal está relacionado con una disrupción del balance entre los macrófagos M1 proinflamatorios y los macrófagos M2 antiinflamatorios con una reducción de los últimos, los cuales median la reparación celular atenuando el estrés oxidativo. ${ }^{135,136}$

En algunos pacientes, la Gp puede considerarse un desorden gastrointestinal autoinmune con la presencia de niveles elevados de autoanticuerpos que pueden desencadenar inflamación y dismotilidad gastrointestinal. En cerca de un 35\% de los pacientes con Gp, se encontró una ganglionitis mientérica relacionada con los linfocitos T. ${ }^{137} \mathrm{~A}$ su vez, en un grupo de pacientes con Gp, se evidenció la presencia de anticuerpos antiglutamato decarboxilasa con una buena respuesta a la administración de la inmunoglobulina endovenosa, el micofenolato o la metilprednisolona. ${ }^{138}$

Más allá de las alteraciones neuromusculares gástricas, los datos recientes sugieren que la dismotilidad en el intestino delgado podría cumplir un rol en la patogenia de la Gp. En un estudio, se identificó dismotilidad entérica en todos los pacientes que presentaban VGR y la severidad de los síntomas se asoció con la misma, no así con el VGR. ${ }^{139}$

Es importante tener en cuenta que, aunque menos estudiados, algunos síntomas de la Gp pueden reflejar anormalidades en la función sensorial gástrica de forma similar a la DF. En los pacientes con síntomas de Gp, se encontró que los mismos no siempre se correlacionan con la severidad en el retraso del VG. ${ }^{140}$ En la Gp idiopática y en la diabética, se ha demostrado que la hipersensibilidad a la distensión gástrica y la alteración de la acomodación gástrica empeoran los síntomas. ${ }^{141,142}$

$\mathrm{Al}$ igual que en la $\mathrm{DF}$, los síntomas de la Gp podrían reflejar un procesamiento anormal de las sensaciones viscerales en el SNC. ${ }^{143}$ En un estudio, se demostró una disminución significativa de la red de conectividad insular bilateral en los pacientes con Gp y náuseas, sugiriendo una vía potencial a través de la cual pueden ser moduladas estas últimas. ${ }^{144}$

\section{Gastroparesia diabética}

La DM es la enfermedad sistémica que más se asocia con la Gp. El VGR es más pronunciado en los pacientes con DM tipo $1 .{ }^{145} \mathrm{La}$ incidencia a 10 años es mayor en la DM tipo $1(5,2 \%)$ que en la tipo $2(1 \%) .{ }^{146} \mathrm{Su}$ fisiopatología es multifactorial con la participación de la hiperglucemia y de la denervación vagal extrínseca y entérica intrínseca. ${ }^{130}$

La hiperglucemia aguda ha mostrado un efecto inhibitorio en el VG mediado por el sistema nervioso entérico, el vago y el descenso de los niveles de motilina. ${ }^{147-148}$

No está claro si la hiperglucemia crónica tiene un efecto en el VG. ${ }^{149,} 150$

\section{Gastroparesia posquirúrgica}

Generalmente causada por la injuria o el atrapamiento del nervio vago, representa la tercera causa más común de Gp. ${ }^{129}$ Se observa con mayor frecuencia luego de la cirugía antirreflujo y bariátrica. La injuria no intencionada del nervio vago ocurre en un 10 a $42 \%$ de las funduplicaturas. ${ }^{151}$ Un estudio que comparó la distensibilidad pilórica con sonda de imagen luminal funcional (FLIP, por su sigla en inglés), luego de diferentes cirugías, demostró que la mayor reducción de la distensibilidad pilórica ocurrió de forma posterior a la cirugía antirreflujo y a la esofaguectomía. ${ }^{152}$ Sin embargo, se ha reportado que los síntomas de estasis gástrica persisten solo en una minoría de los pacientes al año de una funduplicatura de Nissen. ${ }^{153}$

\section{Gastroparesia secundaria a fármacos}

Representa la segunda causa de Gp iatrogénica después de la posquirúrgica. La Gp se asocia con el tratamiento concomitante con los analgésicos opioides, los agentes anticolinérgicos y las drogas neuromoduladoras centrales como los antidepresivos. ${ }^{145,154,155}$

Los agonistas opioides $\mu$ estimulan el tono del esfínter pilórico e inhiben la contractilidad antral con el consecuente retraso del VG. ${ }^{156}$ Incluyen la codeína, la morfina y la oxicodona, ${ }^{157,158}$ observándose un menor efecto con el tramadol. ${ }^{159}$

Los agentes hipoglucemiantes como los análogos de la amilina (pramlintida) y los análogos o agonistas del péptido 1 similar a glucagón (GLP1) (liraglutida y exenatida) pueden retrasar el VG. ${ }^{160}$ En contraste, los inhibidores de la dipeptidil peptidasa IV, como la vildagliptina y la sitagliptina, no se asocian con la Gp. ${ }^{161}$

\section{Gastroparesia posviral}

De forma poco habitual, puede observarse un VG anormal luego de una infección viral aguda autolimitada, especialmente en las mujeres de edad media. ${ }^{162}$ Usualmente, la Gp posviral presenta un buen pronóstico y con fre- 
cuencia mejora dentro del año de seguimiento. ${ }^{163,164}$ Sin embargo, una minoría de los pacientes con Gp asociada a infecciones virales específicas como Epstein-Barr, Norovirus, Herpes virus y Citomegalovirus pueden desarrollar una disfunción autonómica, presentando un peor pronóstico con una resolución más lenta de los síntomas. ${ }^{131}$

\section{Otras causas de gastroparesia}

Los desórdenes del músculo liso infiltrativos (esclerodermia, amiloidosis) o degenerativos (miopatía de víscera hueca, citopatía mitocondrial) pueden ser causas de la Gp. Las enfermedades miopáticas se asocian con una afectación más generalizada del tracto gastrointestinal. ${ }^{130}$ En la esclerodermia, la Gp se relaciona con depósito submucoso de colágeno, reemplazo del músculo liso por tejido fibroso y alteraciones ultraestructurales de las fibras nerviosas. ${ }^{165}$

\section{Diagnóstico}

Una historia clínica detallada debe ayudar a descartar enfermedades orgánicas como DM, colagenopatías, miopatías, causas de daño del nervio vago como la cirugía abdominal o torácica ${ }^{166,167}$ y procedimientos como la ablación cardíaca por fibrilación auricular. ${ }^{168}$ Es importante interrogar sobre la presencia de estreñimiento, trastornos de la defecación, ${ }^{169,}, 170$ síntomas neurológicos autonómicos, ${ }^{166}$ consumo de fármacos que pueden retrasar el $V^{171,172}$ y abuso de cannabinoides como la marihuana. También, es útil evaluar la pérdida de peso no intencionada para determinar la gravedad del trastorno.

El examen físico incluye la evaluación del estado de hidratación y nutrición, la presencia de dolor y distensión abdominal, pudiéndose detectar signos sugestivos de colagenopatías.

La evaluación inicial consta de pruebas de laboratorio de rutina, TSH, evaluación de los marcadores nutricionales y serológicos en los pacientes con bajo peso y de anticuerpos específicos ante la sospecha de colagenopatías. ${ }^{173}$

\section{Videoendoscopía digestiva alta}

Está indicada para excluir la presencia de una obstrucción mecánica. A su vez, permite visualizar el contenido gástrico en ayunas. De forma ocasional, se pueden encontrar bezoares (restos sólidos no digeribles organizados) en los pacientes con atonía gástrica posquirúrgica. En contraste, el hallazgo de restos de comida no organizados retenidos en el estómago es frecuente y no se debe asumir que se debe a la presencia de Gp. Coleski y col. evaluaron, mediante una gammagrafía, a los pacientes que tenían retención de alimentos en la VEDA sin evidencia de una obstrucción mecánica y encontraron que el $74 \%$ presen- taba un VGR. ${ }^{174} \mathrm{El}$ hallazgo de alimentos, en los pacientes con un VG normal, puede estar relacionado con el incumplimiento del ayuno recomendado o, posiblemente, con la variabilidad interindividual en la frecuencia del complejo motor migratorio. ${ }^{175}$

Si la VEDA no revela la causa de los síntomas, se pueden realizar estudios funcionales para medir el VG.

\section{Gammagrafía para medición de vaciamiento gástrico}

Es el gold standard actual para establecer el diagnóstico de Gp. ${ }^{176,177}$ Luego de una noche de ayuno, se les ofrece a los pacientes una comida sólida baja en grasas marcada con tecnecio $99 \mathrm{~m} .{ }^{178}$ Se les realizan imágenes a las 0,1 , 2 y 4 horas para evaluar el tránsito a través del tracto gastrointestinal superior. Una retención gástrica superior al $10 \%$ a las 4 horas indica un VGR. ${ }^{126}$

La técnica tiene varias limitaciones: a) el contenido calórico y de grasas de la comida sugerida es bajo y no reproduce una comida normal, por lo que probablemente subestima la prevalencia real de la Gp, aunque tiene la ventaja de reducir los falsos positivos; b) debido a la exposición a la radiación, debe utilizarse con precaución en las mujeres en edad fértil y no es una técnica ideal para monitorear la evolución clínica o los efectos del tratamiento; c) existen escasos centros de medicina nuclear en el mundo; y d) es un estudio prolongado y costoso. ${ }^{179}$

\section{Prueba del aliento con carbono 13}

Ha sido validada como una alternativa a la gammagrafía de vaciamiento gástrico (GVG). Esta prueba incorpora un isótopo estable, el carbono $13\left({ }^{13} \mathrm{C}\right)$, en un sustrato como el ácido octanoico o la espirulina. Es un método no invasivo, fácil de realizar, con un costo similar a la gammagrafía, que no expone a radiación ionizante; pudiendo utilizarse en mujeres embarazadas o en período de lactancia y en niños. Se lleva a cabo durante un período de 4 horas, después de un ayuno de 8 . Luego de recolectar una muestra de aire espirado basal, los pacientes ingieren una comida que contiene el sustrato mencionado y se toman muestras adicionales cada 30 minutos. A medida que la comida se vacía del estómago, el sustrato se somete a la digestión, la absorción y el metabolismo para producir ${ }^{13} \mathrm{CO}_{2}$, el cual se elimina en el aire espirado. La acumulación de ${ }^{13} \mathrm{CO}_{2}$, en la respiración, refleja la velocidad del VG. ${ }^{180,}{ }^{181}$ Los factores que pueden influir en los resultados de la prueba son cambios en la excreción endógena del $\mathrm{CO}_{2}$ causados por la actividad física, la malabsorción, la insuficiencia pancreática exocrina, la insuficiencia cardíaca y las enfermedades pulmonares o hepáticas. ${ }^{130,182}$ 


\section{Cápsula de motilidad inalámbrica (SmartPill@)}

Ha sido aprobada por la FDA para la evaluación del VG y del tránsito colónico. Mide el $\mathrm{pH}$, la temperatura y la presión en todo el tracto gastrointestinal. ${ }^{183} \mathrm{La}$ finalización del VG se demuestra por un cambio abrupto en el $\mathrm{pH}$ en el rango alcalino debido al paso de la cápsula al duodeno. El VG por cápsula de motilidad inalámbrica tuvo una correlación moderada con la GVG. ${ }^{184}$

Otras técnicas para medir el VG son la RMN y la ecografía, las cuales se han utilizado generalmente con fines de investigación. ${ }^{185,186}$

Actualmente, la electrogastrografía y la manometría antroduodenal no son utilizadas en la práctica clínica. ${ }^{187}$

\section{Tratamiento}

Es razonable recomendar a los pacientes con Gp que eviten las comidas abundantes con un elevado contenido de grasas y fibras, ya que tienden a retrasar el VG. ${ }^{188}$ Debido a que el VG de líquidos con frecuencia está conservado en los pacientes con Gp, se sugiere aumentar la ingesta de nutrientes en forma líquida. ${ }^{189}$ Aunque estas sugerencias se basan en consideraciones fisiopatológicas, no han sido validadas en estudios apropiados.

Más del 60\% de los pacientes con Gp tienen dietas hipocalóricas y presentan deficiencias en vitaminas y minerales. ${ }^{190}$ Por lo tanto, es importante que reciban evaluación y asesoramiento nutricional.

En los pacientes diabéticos, se sugiere un buen control glucémico, a pesar de que no se ha establecido su beneficio a largo plazo en la normalización del VG ni en los síntomas. ${ }^{191}$

\section{Proquinéticos y antieméticos}

La metoclopramida, un antagonista del receptor de dopamina-2 (D2) y agonista parcial del receptor 5-hidroxitriptamina tipo 4 (5-HT4), ejerce efectos proquinéticos y antieméticos. Es el único fármaco aprobado por la FDA para el tratamiento de la Gp, con una restricción de su uso a 12 semanas. Dado que atraviesa la barrera hematoencefálica, puede provocar efectos centrales como ansiedad, depresión, temblores y otros efectos secundarios extrapiramidales más graves que incluyen la discinesia tardía. ${ }^{192-194}$

La eritromicina funciona como agonista de los receptores de motilina y produce una mejoría del VG en los pacientes con $\mathrm{Gp} .{ }^{195} \mathrm{~A}$ pesar de que su efecto gastrocinético es reconocido, hay muy poca evidencia sobre su efecto en los síntomas de los pacientes con Gp. En una revisión sistemática, se evaluó 5 ensayos clínicos no controlados que informaron una mejoría sintomática en el $43 \%$ de los pacientes tratados con este medicamento vía oral $(250 \mathrm{mg}$
3 veces por día en su mayoría) por 2 a 4 semanas. ${ }^{196} \mathrm{La}$ eritromicina puede interactuar con otros fármacos metabolizados por el citocromo P450 3A4, puede producir la prolongación del intervalo QT y tiene la posibilidad de inducir una resistencia bacteriana a los antibióticos ${ }^{126}$ Su respuesta clínica desciende después de 4 semanas de tratamiento, debido a la regulación hacia abajo de los receptores de motilina. ${ }^{197}$

Un abordaje aceptable para contrarrestar los efectos adversos de la metoclopramida y la taquifilaxia de la eritromicina es utilizarlas de forma alterna, en la menor dosis que sea efectiva. Ambas están disponibles en formulación líquida, la cual se prefiere en los pacientes con Gp. ${ }^{126}$

El camicinal, otro agonista de los receptores de motilina, mejoró el VG en pacientes con Gp diabética sin disminución de la respuesta, luego de 28 días de tratamiento. ${ }^{198}$

La prucaloprida es un agonista selectivo del receptor 5-HT4 que ejerce un efecto enterocinético y está aprobado para el tratamiento de la constipación crónica en varios países. Tiene un perfil de seguridad favorable y no afecta el intervalo QT. ${ }^{199,}{ }^{200}$ Recientemente, un ensayo cruzado controlado con placebo evaluó su eficacia en un pequeño número de pacientes con Gp idiopática $(\mathrm{n}=28)$ y diabética $(n=6)$ mostrando una mejoría significativa de los síntomas y de la tasa de VG a sólidos. No se encontró una correlación entre ambos hallazgos, ${ }^{201}$ por lo que se necesitan estudios adicionales para identificar el mecanismo subyacente a la mejoría de los síntomas.

El velusetrag, un potente agonista selectivo del receptor 5-HT4, se encuentra en investigación para el tratamiento de los trastornos de la motilidad gastrointestinal incluida la Gp. En un estudio de fase 2 aleatorizado, doble ciego y cruzado, produjo una mejoría significativa del VG con respecto al placebo $(52 \%$ frente al $5 \%, p=$ 0,002) en 34 pacientes con Gp diabética e idiopática. La mayoría de los efectos adversos fue leve y se los relacionó con la aceleración del tránsito gastrointestinal. ${ }^{202}$

La relamorelina, un agonista selectivo del receptor de la grelina que se administra en forma subcutánea, se encuentra en investigación para el tratamiento de la Gp diabética. Se la evaluó en 2 ensayos de fase 2 controlados con placebo demostrando una mejoría significativa en los síntomas y en el VG. Un 15\% de los pacientes tuvo una hiperglucemia dosis-dependiente como efecto adverso. ${ }^{203,204}$ Actualmente, el fármaco está siendo evaluado en ensayos de fase 3.

El aprepipant, un antagonista del receptor de neuroquinina NK1, aprobado por la FDA para vómitos por quimioterapia o posquirúrgicos, demostró una mejoría 
sintomática y de la acomodación gástrica en los pacientes con Gp. ${ }^{205,206}$

Algunos cannabinoides sintéticos (ej. dronabinol y nabilone) que se utilizan para el tratamiento de náuseas y vómitos por quimioterapia se encuentran en investigación en la $\mathrm{Gp}$, aunque su utilidad es aún controversial. $^{207}$

\section{Neuromoduladores}

A diferencia de lo que ocurre en la DF, la nortriptilina no demostró mejoría de los síntomas en un ECA de 65 pacientes con Gp idiopática. ${ }^{208}$

La mirtazapina mejoró, de forma significativa, las náuseas y los vómitos en un ensayo no controlado con placebo que evaluó a 30 pacientes con Gp refractaria a otros tratamientos. A su vez, mejoró la hiporexia y aumentó la capacidad de terminar una comida de tamaño normal. Los efectos adversos más frecuentes fueron la somnolencia y la fatiga. ${ }^{209}$ Se necesitan ECA para evaluar esta indicación para mirtazapina y determinar posibles predictores de respuesta.

La levosulpirida, un agente antipsicótico con actividad antidopaminérgica y serotoninérgica, mostró una mejoría de los síntomas en los pacientes con Gp diabética ${ }^{210}$ e idiopática, ${ }^{211}$ sin cambios en el VG.

\section{Estimulación eléctrica gástrica}

El tratamiento no farmacológico se reserva para los casos graves de Gp con síntomas refractarios a las drogas vigentes. ${ }^{212}$

La estimulación eléctrica gástrica (EEG) consiste en dos electrodos que se colocan, mediante laparoscopía o laparotomía, en la capa muscular de la curvatura mayor del estómago; y un marcapasos subcutáneo en la pared abdominal. Antes de colocar el dispositivo permanente, se puede realizar una estimulación eléctrica temporal para determinar la respuesta. Este procedimiento fue aprobado por la FDA para el tratamiento de la Gp idiopática y diabética, aunque su uso ha sido controvertido. Una revisión sistemática y metanálisis evaluó 5 ECA sin encontrar un beneficio clínico luego de la intervención. ${ }^{213}$ Un estudio reciente describe una experiencia multicéntrica en EEG a partir de una base de datos prospectiva de más de 300 pacientes con síntomas de Gp, con y sin VGR. El trabajo mostró que los pacientes que se sometieron a la EEG experimentaron mejoría significativa de las náuseas (RR 1,31; IC 95\% 1,03-1,67; $p=0,04)$. $^{214}$ Un ECA reciente con diseño cruzado demostró que la EEG redujo la frecuencia de náuseas y vómitos (Score de vómitos ON 2,3 $\pm 1,7$ vs. OFF $1,9 \pm 1,7 ; p=0,01$ ) en pacientes con $\mathrm{Gp}$, a pesar de que no se observó una mejoría significativa en el VG. ${ }^{215}$

\section{Procedimientos endoscópicos}

Como se mencionó anteriormente, la disfunción pilórica contribuye a la fisiopatología de la Gp. En la última década, el advenimiento de la planimetría por impedancia, mediante el uso de FLIP, permitió evaluar la distensibilidad de diferentes segmentos del tracto gastrointestinal. Recientemente, se demostró que la distensibilidad pilórica disminuida se correlaciona con los síntomas de la Gp, aunque hubo discordancia en cuanto a la correlación con el VGR. Esta técnica podría ser útil para identificar a los pacientes con $\mathrm{Gp}$ que pueden beneficiarse de terapias dirigidas al píloro. ${ }^{216,217}$

Los estudios iniciales no controlados sugirieron una buena respuesta a la inyección de toxina botulínica en el músculo pilórico. ${ }^{218,219}$ Sin embargo, dos investigaciones controladas posteriores no demostraron superioridad en comparación con la inyección de solución salina. $^{220,221}$

De forma reciente, se ha desarrollado la miotomía endoscópica peroral gástrica (G-POEM, por su sigla en inglés) como un abordaje terapéutico novedoso. Esta técnica mínimamente invasiva emplea los principios de la miotomía endoscópica peroral (POEM) esofágica y fue realizada por primera vez en humanos por Khashab y col. en 2013.222 Una revisión sistemática reciente que evaluó su seguridad y eficacia en el tratamiento de la Gp encontró tasas de respuesta clínica del 73 al 86\% con tasas bajas de complicaciones (0-6,7\%). ${ }^{223} \mathrm{Un}$ metanálisis reciente incluyó a 7 estudios con un total de 196 pacientes con Gp refractaria, mostrando una mejoría significativa de los síntomas a los 5 días y del VG a los 2-3 meses del procedimiento. Se reportaron efectos adversos en el 6\% de los pacientes, siendo el más frecuente el capnoperitoneo, el cual se manejó satisfactoriamente mediante descompresión con aguja. ${ }^{224}$ La G-POEM tuvo éxito clínico en el tratamiento de la Gp refractaria en otra revisión sistemática reciente con metanálisis, con resultados clínicos similares a los obtenidos con la piloroplastia quirúrgica. ${ }^{225}$

A pesar de que estos resultados iniciales son prometedores, es necesario tener precaución al interpretarlos debido a que se basan en estudios no controlados con seguimiento a corto plazo. Se requieren ensayos controlados de mayor tamaño para seleccionar el subgrupo de pacientes con disfunción pilórica que se podría beneficiar de esta técnica al igual que para determinar su eficacia a largo plazo. 


\section{Conclusión}

Esta revisión abordó aspectos importantes en el conocimiento actual sobre fisiopatología, diagnóstico y tratamiento de la DF y la Gp. Existe una superposición significativa entre ambos trastornos, los cuales se consideran como parte de un espectro de disfunción sensitivo-motora gastroduodenal. Las investigaciones recientes y en curso se centran en nuevos mecanismos fisiopatológicos y en novedosos abordajes terapéuticos que permitirán mejorar el manejo de los pacientes, especialmente de aquellos con síntomas refractarios a las terapias vigentes.

\section{Aviso de derechos de autor}

\section{(C) 2021 Acta Gastroenterológica (C) $\$($ (O) Latinoamericana. Este es un artículo BY NC SA de acceso abierto publicado bajo los términos de la Licencia Creative Commons Attribution (CC BY-NC-SA 4.0), la cual permite el uso, la distribución y la reproducción de forma no comercial, siempre que se cite al autor y la fuente original.}

Cite este artículo como: Manresa MM, Carboné PC, Diez G. Dispepsia funcional y gastroparesia: ¿dos entidades superpuestas? De la fisiopatología a la terapéutica. Acta Gastroenterol Latinoam 2021;51(2):143-62. https://doi. org/10.52787/bvbw 1724

\section{Referencias}

1. Lacy BE. Functional dyspepsia and gastroparesis: one disease or two? Am J Gastroenterol. 2012;107(11):1615-20.

2. Cangemi DJ, Lacy BE. Gastroparesis and functional dyspepsia: different diseases or different ends of the spectrum? Curr Opin Gastroenterol. 2020;36(6):509-17.

3. Parkman HP, Hasler WL, Fisher RS; American Gastroenterological Association. American Gastroenterological Association technical review on the diagnosis and treatment of gastroparesis. Gastroenterology. 2004;127(5):1592-622.

4. Van Lelyveld N, Schipper M, Samsom M. Lack of Relationship Between Chronic Upper Abdominal Symptoms and Gastric Function in Functional Dyspepsia. Digestive Diseases and Sciences. 2008;53(5):1223-30.

5. Karamanolis G, Caenepeel P, Arts J, Tack J. Association of the Predominant Symptom With Clinical Characteristics and Pathophysiological Mechanisms in Functional Dyspepsia. Gastroenterology. 2006;130(2):296-303.

6. Fischler B, Vandenberghe J, Persoons P, De Gucht V, Broekaert D, Luyckx K, Tack J. Evidence-based subtypes in functional dyspepsia with confirmatory factor analysis: Psychosocial and physiopathological correlates. Gastroenterology. 2001;120:A51-2.
7. Van Oudenhove L, Holvoet L, Vandenberghe J, Vos R, Tack J. Do we have an alternative for the Rome III gastroduodenal symptom-based subgroups in functional gastroduodenal disorders? A cluster analysis approach. Neurogastroenterol Motil. 2011;23(8):730-8.

8. Tack J, Talley NJ, Camilleri M, Holtmann G, Hu P, Malagelada JR, Stanghellini V. Functional Gastroduodenal Disorders. Gastroenterology. 2006;130(5):1466-79.

9. Stanghellini V, Chan FKL, Hasler WL, Malagelada JR, Suzuki H, Tack J, Talley NJ. Gastroduodenal Disorders. Gastroenterology. 2016;150(6):1380-92.

10. Aziz I, Palsson OS, Törnblom H, Sperber AD, Whitehead WE, Simrén M. Epidemiology, clinical characteristics, and associations for symptom-based Rome IV functional dyspepsia in adults in the USA, Canada, and the UK: a cross-sectional population-based study. The Lancet Gastroenterology \& Hepatology. 2018;3(4):252-62.

11. Ford AC, Marwaha A, Sood R, Moayyedi P. Global prevalence of, and risk factors for, uninvestigated dyspepsia: a meta-analysis. Gut. 2015;64(7):1049-57.

12. Sugano K, Tack J, Kuipers EJ, Graham DY, El-Omar EM, Miura S, Haruma K, Asaka M, Uemura N, Malfertheiner P, faculty members of Kyoto Global Consensus Conference. Kyoto global consensus report on Helicobacter pylori gastritis. Gut. 2015;64:1353-67.

13. Fang YJ, Liou JM, Chen CC, Lee JY, Hsu YC, Chen MJ, Tseng $\mathrm{PH}$, Chen CC, Chang CY, Yang TH, Chang WH, Wu JY, Wang HP, Luo JC, Lin JT, Shun CT, Wu MS; Taiwan Gastrointestinal Disease and Helicobacter Consortium. Distinct aetiopathogenesis in subgroups of functional dyspepsia according to the Rome III criteria. Gut. 2015;64:1517-28.

14. Hsu YC, Liou JM, Liao SC, Yang TH, Wu HT, Hsu WL, Lin HJ, Wang HP, Wu MS. Psychopathology and personality trait in subgroups of functional dyspepsia based on Rome III criteria. Am J Gastroenterol. 2009;104(10):2534-42.

15. Aro P, Talley NJ, Ronkainen J, Storskrubb T, Vieth M, Johansson SE, Bolling-Sternevald E, Agréus L. Anxiety is associated with uninvestigated and functional dyspepsia (Rome III criteria) in a Swedish population-based study. Gastroenterology. 2009; 137:94-100.

16. Gracie DJ, Bercik P, Morgan DG, Bolino C, Pintos-Sanchez MI, Moayyedi P, Ford AC. No increase in prevalence of somatization in functional vs organic dyspepsia: a cross-sectional survey. Neurogastroenterol Motil. 2015;27(7):1024-31.

17. Aro P, Talley NJ, Agréus L, Johansson SE, Bolling-Sternevald E, Storskrubb T, Ronkainen J. Functional dyspepsia impairs quality of life in the adult population. Aliment Pharmacol Ther. 2011;33(11):1215-24.

18. Perveen I, Parvin R, Saha M, Bari MS, Huda MN, Ghosh Mk. Prevalence of Irritable Bowel Syndrome (IBS), Migraine and Co-Existing IBS-Migraine in Medical Students. J Clin Diagn Res. 2016;10(11):OC09-13.

19. Locke GR, Zinsmeister AR, Fett SL, Melton LJ, Talley NJ. Overlap of gastrointestinal symptom complexes in a US community. Neurogastroenterol Motil. 2005;17:29-34.

20. Talley NJ. Editorial: Moving Away From Focussing on Gastric Pathophysiology in Functional Dyspepsia: New Insights and Therapeutic Implications. The American journal of gastroenterology. 2017;112:141-4. 
21. Carbone F, Tack J. Gastroduodenal mechanisms underlying functional gastric disorders. Dig Dis. 2014;32(3):222-9.

22. Vanheel H, Carbone F, Valvekens L, Simren M, Tornblom H, Vanuytsel T, Van Oudenhove L, Tack J. Pathophysiological Abnormalities in Functional Dyspepsia Subgroups According to the Rome III Criteria. Am J Gastroenterol. 2017;112:132-40.

23. Delgado-Aros S, Camilleri M, Cremonini F, Ferber I, Stephens D, Burton DD. Contributions of gastric volumes and gastric emptying to meal size and postmeal symptoms in functional dyspepsia. Gastroenterology. 2004;127(6):1685-94.

24. Ardila-Hani A, Arabyan M, Waxman A, Ih G, Berel D, Pimentel M, Conklin J, Soffer EE. Severity of dyspeptic symptoms correlates with delayed and early variables of gastric emptying. Dig Dis Sci. 2013;58(2):478-87.

25. Haag S, Talley NJ, Holtmann G. Symptom patterns in functional dyspepsia and irritable bowel syndrome: relationship to disturbances in gastric emptying and response to a nutrient challenge in consulters and non-consulters. Gut. 2004;53:1445-51.

26. Talley NJ, Locke GR, Lahr BD, Zinsmeister AR, Tougas G, Ligozio G, Rojavin MA, Tack J. Functional dyspepsia, delayed gastric emptying, and impaired quality of life. Gut. 2006;55(7):933-9.

27. Karamanolis G, Caenepeel P, Arts J, Tack J. Association of the predominant symptom with clinical characteristics and pathophysiological mechanisms in functional dyspepsia. Gastroenterology. 2006;130(2):296-303.

28. Jian R, Ducrot F, Ruskone A, Chaussade S, Rambaud JC, Modigliani R, Rain JD, Bernier JJ. Symptomatic, radionuclide and therapeutic assessment of chronic idiopathic dyspepsia. Digestive Diseases and Sciences. 1989;34:657-64.

29. Vijayvargiya P, Camilleri M, Chedid V, Mandawat A, Erwin PJ, Murad MH. Effects of Promotility Agents on Gastric Emptying and Symptoms: A Systematic Review and Meta-analysis. Gastroenterology. 2019;156(6):1650-60.

30. Park S-Y, Acosta A, Camilleri M, Burton D, Harmsen WS, Fox J, Szarka LA. Gastric Motor Dysfunction in Patients With Functional Gastroduodenal Symptoms. Am J Gastroenterol. 2017;112(11):1689-99.

31. Kim DY, Delgado-Aros S, Camilleri M, Samsom M, Murray JA, O'Connor MK, Brinkmann BH, Stephens DA, Lighvani SS, Burton DD. Noninvasive measurement of gastric accommodation in patients with idiopathic nonulcer dyspepsia. Am J Gastroenterol. 2001;96(11):3099-105.

32. Caldarella MP, Azpiroz F, Malagelada J-R. Antro-fundic dysfunctions in functional dyspepsia. Gastroenterology. 2003;124:1220-9.

33. Bortolotti M, Bolondi L, Santi V, Sarti P, Brunelli F, Barbara L. Patterns of Gastric Emptying in Dysmotility-Like Dyspepsia. Scandinavian Journal of Gastroenterology. 1995;30(5):408-10.

34. Mertz H, Fullerton S, Naliboff B, Mayer E. Symptoms and visceral perception in severe functional and organic dyspepsia. Gut. 1998;42(6):814-22.

35. Holtmann G, Goebell H, Talley J. Impaired small intestinal peristaltic reflexes and sensory thresholds are independent functional disturbances in patients with chronic unexplained dyspepsia. Am J Gastroenterol. 1996;91:485-91.

36. Greydanus MP, Vassallo M, Camilleri M, Nelson DK, Hanson RB, Thomforde GM. Neurohormonal factors in functional dyspepsia: insights on pathophysiological mechanisms. Gastroenterology. 1991;100:1311-8.
37. Bouin M, Lupien F, Riberdy M, Boivin M, Plourde V, Poitras P. Intolerance to visceral distension in functional dyspepsia or irritable bowel syndrome: an organ specific defect or a pan intestinal dysregulation? Neurogastroenterol Motil. 2004;16(3):311-4.

38. Simrén M, Törnblom H, Palsson OS, van Tilburg MAL, Van Oudenhove L, Tack J, Whitehead WE. Visceral hypersensitivity is associated with GI symptom severity in functional GI disorders: consistent findings from five different patient cohorts. Gut. 2018;67(2):255-62.

39. Tack J, Caenepeel P, Fischler B, Piessevaux H, Janssens J. Symptoms associated with hypersensitivity to gastric distention in functional dyspepsia. Gastroenterology. 2001;121(3):526-35.

40. Farré R, Vanheel H, Vanuytsel T, Masaoka T, Törnblom H, Simrén M, Van Oudenhove L, Tack JF. In Functional Dyspepsia, Hypersensitivity to Postprandial Distention Correlates With Meal-Related Symptom Severity. Gastroenterology. 2013;145(3):566-73.

41. Oshima T, Okugawa T, Tomita T, Sakurai J, Toyoshima F, Watari J, Yamaguchi K, Fujimoto K, Adachi K, Kinoshita Y, Kusunoki H, Haruma K, Miwa H. Generation of dyspeptic symptoms by direct acid and water infusion into the stomachs of functional dyspepsia patients and healthy subjects. Aliment Pharmacol Ther. 2012;35:175-82.

42. Samsom M, Verhagen MA, vanBerge Henegouwen GP, Smout AJ. Abnormal clearance of exogenous acid and increased acid sensitivity of the proximal duodenum in dyspeptic patients. Gastroenterology. 1999;116(3):515-20.

43. Lee KJ, Vos R, Janssens J, Tack J. Influence of duodenal acidification on the sensorimotor function of the proximal stomach in humans. Am J Physiol Gastrointest Liver Physiol. 2004;286:G278-84.

44. Vanuytsel T, Karamanolis G, Vos R, Van Oudenhove L, Farré $\mathrm{R}$, Tack J. Role of duodenal mucosal nerve endings in the acid-induced duodenogastric sensorimotor reflex: effect of benzocaine in healthy humans. Neurogastroenterol Motil. 2013;25(5):e353-61.

45. Barbera R, Feinle C, Read NW. Nutrient-specific modulation of gastric mechanosensitivity in patients with functional dyspepsia. Dig Dis Sci. 1995;40:1636-41.

46. Vanheel H, Vicario M, Vanuytsel T, Van Oudenhove L, Martinez C, Keita ÅV, Pardon N, Santos J, Söderholm JD, Tack J, Farré R. Impaired duodenal mucosal integrity and low-grade inflammation in functional dyspepsia. Gut. 2014;63:262-71.

47. Walker MM, Aggarwal KR, Shim LS, Bassan M, Kalantar JS, Weltman MD, Jones M, Powell N, Talley NJ. Duodenal eosinophilia and early satiety in functional dyspepsia: Confirmation of a positive association in an Australian cohort. Journal of Gastroenterology and Hepatology. 2014;29:474-9.

48. Talley NJ, Walker MM, Aro P, Ronkainen J, Storskrubb T, Hindley LA, Harmsen WS, Zinsmeister AR, Agréus L. Non-ulcer dyspepsia and duodenal eosinophilia: an adult endoscopic population-based case-control study. Clin Gastroenterol Hepatol. 2007;5(10):1175-83.

49. Futagami S, Shindo T, Kawagoe T, Horie A, Shimpuku M, Gudis K, Iwakiri K, Itoh T, Sakamoto C. Migration of Eosinophils and CCR2-/CD68-Double Positive Cells Into the Duodenal Mucosa of Patients With Postinfectious Functional Dyspepsia. American Journal of Gastroenterology. 2010;105(8):1835-42. 
50. Walker MM, Salehian SS, Murray CE, Rajendran A, Hoare JM, Negus R, Powell N, Talley NJ. Implications of eosinophilia in the normal duodenal biopsy - an association with allergy and functional dyspepsia. Aliment Pharmacol Ther. 2010;31(11):1229-36.

51. Walker MM, Talley NJ, Prabhakar M, Pennaneac'h CJ, Aro P, Ronkainen J, Storskrubb T, Harmsen WS, Zinsmeister AR, Agreus L. Duodenal mastocytosis, eosinophilia and intraepithelial lymphocytosis as possible disease markers in the irritable bowel syndrome and functional dyspepsia. Aliment Pharmacol Ther. 2009;29(7):765-73.

52. Ronkainen J, Aro P, Walker MM, Agréus L, Johansson SE, Jones $\mathrm{M}$, Talley NJ. Duodenal eosinophilia is associated with functional dyspepsia and new onset gastro-oesophageal reflux disease. Aliment Pharmacol Ther. 2019;50:24-32.

53. Wang X, Li X, Ge W, Huang J, Li G, Cong Y, Li F, Liu Z, Liu Z, Li Y, Yuan H. Quantitative evaluation of duodenal eosinophils and mast cells in adult patients with functional dyspepsia. Ann Diagn Pathol. 2015;19(2):50-6.

54. Du L, Shen J, Kim JJ, Yu Y, Ma L, Dai N. Increased Duodenal Eosinophil Degranulation in Patients with Functional Dyspepsia: A Prospective Study. Sci Rep. 2016;6:34305.

55. Vanheel H, Vicario M, Boesmans W, Vanuytsel T, Salvo-Romero E, Tack J, Farré R. Activation of Eosinophils and Mast Cells in Functional Dyspepsia: an Ultrastructural Evaluation. Sci Rep. 2018;8:5383.

56. Walker MM, Warwick A, Ung C, Talley NJ. The role of eosinophils and mast cells in intestinal functional disease. Curr Gastroenterol Rep. 2011;13(4):323-30.

57. Nakagawa K, Hara K, Fikree A, Siddiqi S, Woodland P, Masamune A, Aziz Q, Sifrim D, Yazaki E. Patients with dyspepsia have impaired mucosal integrity both in the duodenum and jejunum: in vivo assessment of small bowel mucosal integrity using baseline impedance. J Gastroenterol. 2020;55(3):273-80.

58. Cirillo C, Bessissow T, Desmet AS, Vanheel H, Tack J, Berghe PV. Evidence for neuronal and structural changes in submucous ganglia of patients with functional dyspepsia. Am J Gastroenterol. 2015;110(8):1205-15.

59. Liebregts T, Adam B, Bredack C, Gururatsakul M, Pilkington KR, Brierley SM, Blackshaw LA, Gerken G, Talley NJ, Holtmann G. Small bowel homing T cells are associated with symptoms and delayed gastric emptying in functional dyspepsia. Am J Gastroenterol. 2011;106(6):1089-98.

60. Tack J, Demedts I, Dehondt G, Caenepeel P, Fischler B, Zandecki M, Janssens J. Clinical and pathophysiological characteristics of acute-onset functional dyspepsia. Gastroenterology. 2002;122(7):1738-47.

61. Pike BL, Porter CK, Sorrell TJ, Riddle MS. Acute gastroenteritis and the risk of functional dyspepsia: a systematic review and meta-analysis. Am J Gastroenterol. 2013;108(10):1558-63.

62. Futagami S, Itoh T, Sakamoto C. Systematic review with meta-analysis: post-infectious functional dyspepsia. Alimentary Pharmacology \& Therapeutics. 2015;41(2):177-88.

63. Mearin F, Pérez-Oliveras M, Perelló A, Vinyet J, Ibañez A, Coderch J, Perona M. Dyspepsia and irritable bowel syndrome after a Salmonella gastroenteritis outbreak: one-year follow-up cohort study. Gastroenterology. 2005;129:98-104.

64. Dizdar V, Gilja OH, Hausken T. Increased visceral sensitivity in Giardia-induced postinfectious irritable bowel syndrome and functional dyspepsia. Effect of the 5HT3-antagonist ondansetron. Neurogastroenterology \& Motility. 2007;19(12):977-82.
65. Porter CK, Gormley R, Tribble DR, Cash BD, Riddle MS. The Incidence and Gastrointestinal Infectious Risk of Functional Gastrointestinal Disorders in a Healthy US Adult Population. American Journal of Gastroenterology. 2011;106:130-8.

66. Spiller R. Postinfectious functional dyspepsia and postinfectious irritable bowel syndrome: different symptoms but similar risk factors. Gastroenterology. 2010;138:1660-3.

67. Gwee KA, Leong YL, Graham C, McKendrick MW, Collins SM, Walters SJ, Underwood JE, Read NW. The role of psychological and biological factors in postinfective gut dysfunction. Gut. 1999;44(3):400-6.

68. Ohman L, Simrén M. Intestinal Microbiota and its Role in Irritable Bowel Syndrome (IBS). Current Gastroenterology Reports. 2013;15:323-7.

69. Zhong L, Shanahan ER, Raj A, Koloski NA, Fletcher L, Morrison M, Walker MM, Talley NJ, Holtmann G. Dyspepsia and the microbiome: time to focus on the small intestine. Gut. 2017;66(6):1168-9.

70. Igarashi M, Nakae H, Matsuoka T, Takahashi S, Hisada T, Tomita J, Koga Y. Alteration in the gastric microbiota and its restoration by probiotics in patients with functional dyspepsia. BMJ Open Gastroenterol. 2017;4:e000144.

71. Gomes Costa MB, Azeredo IL, Marciano RD, Caldeira LM, Bafutto M. Evaluation of small intestine bacterial overgrowth in patients with functional dyspepsia through $\mathrm{H} 2$ breath test. Arq Gastroenterol. 2012;49(4):279-83.

72. Shimura S, Ishimura N, Mikami H, Okimoto E, Uno G, Tamagawa Y, Aimi M, Oshima N, Sato S, Ishihara S, Kinoshita Y. Small Intestinal Bacterial Overgrowth in Patients with Refractory Functional Gastrointestinal Disorders. J Neurogastroenterol Motil. 2016;22:60-8.

73. Aro P, Talley NJ, Johansson SE, Agréus L, Ronkainen J. Anxiety Is Linked to New-Onset Dyspepsia in the Swedish Population: A 10-Year Follow-up Study. Gastroenterology. 2015;148(5):928-37.

74. Koloski NA, Jones M, Kalantar J, Weltman M, Zaguirre J, Talley NJ. The brain-gut pathway in functional gastrointestinal disorders is bidirectional: a 12-year prospective population-based study. Gut. 2012;61(9):1284-90.

75. Koloski NA, Jones M, Talley NJ. Evidence that independent gutto-brain and brain-to-gut pathways operate in the irritable bowel syndrome and functional dyspepsia: a 1-year population-based prospective study. Aliment Pharmacol Ther. 2016;44:592-600.

76. Vandenberghe J, Dupont P, Van Oudenhove L, Bormans G, Demyttenaere K, Fischler B, Geeraerts B, Janssens J, Tack J. Regional cerebral blood flow during gastric balloon distention in functional dyspepsia. Gastroenterology. 2007;132(5):1684-93.

77. Lee IS, Wang H, Chae Y, Preissl H, Enck P. Functional neuroimaging studies in functional dyspepsia patients: a systematic review. Neurogastroenterol Motil. 2016;28(6):793-805.

78. Chen Y, Wang R, Hou B, Feng F, Fang X, Zhu L, Sun X, Wang Z, Ke M. Regional Brain Activity During Rest and Gastric Water Load in Subtypes of Functional Dyspepsia: A Preliminary Brain Functional Magnetic Resonance Imaging Study. J Neurogastroenterol Motil. 2018;24(2):268-79.

79. Rodiño-Janeiro BK, Alonso-Cotoner C, Pigrau M, Lobo B, Vicario M, Santos J. Role of Corticotropin-releasing Factor in Gastrointestinal Permeability. J Neurogastroenterol Motil. 2015;21:33-50. 
80. Vanuytsel T, van Wanrooy S, Vanheel H, Vanormelingen C, Verschueren S, Houben E, Salim Rasoel S, Tóth J, Holvoet L, Farré R, Van Oudenhove L, Boeckxstaens G, Verbeke K, Tack J. Psychological stress and corticotropin-releasing hormone increase intestinal permeability in humans by a mast cell-dependent mechanism. Gut. 2014;63(8):1293-9.

81. Moayyedi P, Talley NJ, Fennerty MB, Vakil N. Can the Clinical History Distinguish Between Organic and Functional Dyspepsia? JAMA. 2006;295(13):1566-76.

82. Moayyedi PM, Lacy BE, Andrews CN, Enns RA, Howden CW, Vakil N. ACG and CAG Clinical Guideline: Management of Dyspepsia. Am J Gastroenterol. 2017;112:988-1013.

83. Heikkinen M, Räsänen $H$, Färkkilä M. Clinical value of ultrasound in the evaluation of dyspepsia in primary health care. Scandinavian Journal of Gastroenterology. 2005;40(8):980-4.

84. Ford AC, Marwaha A, Lim A, Moayyedi P. What is the prevalence of clinically significant endoscopic findings in subjects with dyspepsia? Systematic review and meta-analysis. Clin Gastroenterol Hepatol. 2010;8(10):830-7.

85. Aguilar Paiz L, Barreda F, Burgos H, Bustos Fernández L, Defilippi C, González JC, Gutiérrez Castrellón P, Hani A, López Colombo A, Leyton JC, Pérez CL, Moreno JM, Olmos J, Oviedo C, Quintero I, Valdovinos MA. Guía Latinoamericana de Dispepsia Funcional. Acta Gastroenterol Latinoam. 2014;44(2):1-80.

86. American Gastroenterological Association. American Gastroenterological Association Institute Guideline on the Role of Upper Gastrointestinal Biopsy to Evaluate Dyspepsia in the Adult Patient in the Absence of Visible Mucosal Lesions: Clinical Decision Support Tool. Gastroenterology. 2015;149(4):1119.

87. Carmona Sánchez R, Gómez Escudero O, Zavala Solares M, Bielsa Fernández MV, Coss Adame E, Hernández Guerrero AI, Huerta Iga F, Icaza Chávez ME, Lira Pedrín MA, Lizárraga López JA, López Colombo A, Noble Lugo A, Pérez Manauta J, Raña Garibay RH, Remes Troche JM, Tamayo JL, Uscanga LF, Zamarripa Dorsey F, Valdovinos Díaz MA, Velarde Ruiz Velasco JA. Consenso mexicano sobre la dispepsia. Revista de Gastroenterología de México. 2017;82:309-27.

88. Ford AC, Mahadeva S, Carbone MF, Lacy BE, Talley NJ. Functional dyspepsia. Lancet. 2020;396(10263):1689-702.

89. Duncanson KR, Talley NJ, Walker MM, Burrows TL. Food and functional dyspepsia: a systematic review. Journal of Human $\mathrm{Nu}$ trition and Dietetics. 2018;31(3):390-407.

90. Moayyedi P, Soo S, Deeks J, Forman D, Mason J, Innes M, Delaney B. Systematic review and economic evaluation of Helicobacter pylori eradication treatment for non-ulcer dyspepsia. Dyspepsia Review Group. BMJ. 2000;321(7262):659-64.

91. Moayyedi P, Soo S, Deeks J, Delaney B, Harris A, Innes M, Oakes R, Wilson S, Roalfe A, Bennett C, Forman D. Eradication of Helicobacter pylori for non-ulcer dyspepsia. Cochrane Database of Systematic Reviews. 2011;19(2):CD002096.

92. Makris N, Barkun A, Crott R, Fallone CA. Cost-effectiveness of alternative approaches in the management of dyspepsia. Int J Technol Assess Health Care. 2003;19(3):446-64.

93. Suzuki H, Moayyedi P. Helicobacter pylori infection in functional dyspepsia. Nat Rev Gastroenterol Hepatol. 2013;10:168-74.

94. Moayyedi P. Helicobacter pylori Eradication for Functional Dyspepsia: What Are We Treating? Archives of Internal Medicine. 2011;171:1936-7.
95. Moayyedi P, Yuan Y, Leontiadis G, CAG Clinical Affairs. Canadian Association of Gastroenterology position statement: hip fracture and proton pump inhibitor therapy-a 2013 update. Can J Gastroenterol. 2013;27(10):593-5.

96. Pinto-Sanchez MI, Yuan Y, Hassan A, Bercik P, Moayyedi P. Proton pump inhibitors for functional dyspepsia. Cochrane Database Syst Rev. 2017;11(11):CD011194.

97. Wauters L, Ceulemans M, Frings D, Lambaerts M, Accarie A, Toth J, Mols R, Augustijns P, De Hertogh G, Van Oudenhove L, Tack J, Vanuytsel T. Proton Pump Inhibitors Reduce Duodenal Eosinophilia, Mast Cells, and Permeability in Patients With Functional Dyspepsia. Gastroenterology. 2021;160(5):1521-31.

98. Ford AC, Luthra P, Tack J, Boeckxstaens GE, Moayyedi P, Talley NJ. Efficacy of psychotropic drugs in functional dyspepsia: systematic review and meta-analysis. Gut. 2017;66(3):411-20.

99. Talley NJ, Locke GR, Saito YA, Almazar AE, Bouras EP, Howden CW, Lacy BE, DiBaise JK, Prather CM, Abraham BP, El-Serag HB, Moayyedi P, Herrick LM, Szarka LA, Camilleri M, Hamilton FA, Schleck CD, Tilkes KE, Zinsmeister AR. Effect of Amitriptyline and Escitalopram on Functional Dyspepsia: A Multicenter, Randomized Controlled Study. Gastroenterology. 2015;149(2):340-9.

100. Tan VPY, Cheung TK, Wong WM, Pang R, Wong BCY. Treatment of functional dyspepsia with sertraline: a double-blind randomized placebo-controlled pilot study. World J Gastroenterol. 2012;18(42):6127-33.

101. Tack J, Ly HG, Carbone F, Vanheel H, Vanuytsel T, Holvoet L, Boeckxstaens G, Caenepeel P, Arts J, Van Oudenhove L. Efficacy of Mirtazapine in Patients With Functional Dyspepsia and Weight Loss. Clin Gastroenterol Hepatol. 2016;14(3):385-92.

102. Jiang SM, Jia L, Liu J, Shi MM, Xu MZ. Beneficial effects of antidepressant mirtazapine in functional dyspepsia patients with weight loss. World J Gastroenterol. 2016;22(22):5260-6.

103. Pittayanon R, Yuan Y, Bollegala NP, Khanna R, Lacy BE, Andrews CN, Leontiadis GI, Moayyedi P. Prokinetics for Functional Dyspepsia: A Systematic Review and Meta-Analysis of Randomized Control Trials. Am J Gastroenterol. 2019;114(2):233-43.

104. Matsueda K, Hongo M, Tack J, Saito Y, Kato H. A placebo-controlled trial of acotiamide for meal-related symptoms of functional dyspepsia. Gut. 2012;61(6):821-8.

105. Marciniak TA, Serebruany V. Should We Use Tegaserod for Irritable Bowel Syndrome? Am J Ther. 2019;26(3):e417-20.

106. Madia VN, Messore A, Saccoliti F, Tudino V, De Leo A, De Vita D, Bortolami M, Scipione L, Pindinello I, Costi R, Di Santo R. Tegaserod for the Treatment of Irritable Bowel Syndrome. Antiinflamm Antiallergy Agents Med Chem. 2020;19(4):342-69.

107. Bekhti A, Rutgeerts L. Domperidone in the treatment of functional dyspepsia in patients with delayed gastric emptying. Postgrad Med J. 1979;55(1):30-2.

108. Chey WY, You CH, Ange DA. Open and double-blind clinical trials of domperidone in patients with unexplained nausea, vomiting, abdominal bloating and early satiety. Gastroenterology. 1982;82:1033.

109. Davis RH, Clench MH, Mathias JR. Effects of domperidone in patients with chronic unexplained upper gastrointestinal symptoms: a double-blind, placebo-controlled study. Dig Dis Sci. 1988;33:1505-11. 
110. Haarmann K, Lebkuchner F, Widmann A, Kief W, Esslinger M. A double-blind study of domperidone in the symptomatic treatment of chronic post-prandial upper gastrointestinal distress. Postgrad Med J. 1979;55(1):24-7.

111. Van de Mierop L, Rutgeerts L, Van den Langenbergh B, Staessen A. Oral Domperidone in Chronic Postprandial Dyspepsia. A double-blind placebo-controlled evaluation. Digestion. 1979;19(4):244-50.

112. Van G, Ganse V, Damme V. Chronic dyspepsia: double blind treatment with domperidone or a placebo. A multicentre therapeutic evaluation. Curr Therapeut Res. 1978;23(6):695-701.

113. Van Outryve M, Lauwers W, Verbeke S. Domperidone for the symptomatic treatment of chronic post-prandial nausea and vomiting. Postgrad Med J. 1979;55(1):33-5.

114. Zhang J, Wu HM, Wang X, Xie J, Li X, Ma J, Wang F, Tang X. Efficacy of prebiotics and probiotics for functional dyspepsia: A systematic review and meta-analysis. Medicine. 2020;99:e19107.

115. Navarro-Rodriguez T, Silva FM, Barbuti RC, Mattar R, Prado Moraes-Filho J, Nogueira de Oliveira M, Bogsan CS, Chinzon D, Eisig JN. Association of a probiotic to a Helicobacter pylori eradication regimen does not increase efficacy or decreases the adverse effects of the treatment: a prospective, randomized, double-blind, placebo-controlled study. BMC Gastroenterol. 2013;13:56.

116. Takagi A, Yanagi H, Ozawa H, Uemura N, Nakajima S, Inoue K, Kawai T, Ohtsu T, Koga Y. Effects of Lactobacillus gasseri OLL2716 on Helicobacter pylori-Associated Dyspepsia: A Multicenter Randomized Double-Blind Controlled Trial. Gastroenterol Res Pract. 2016;2016:7490452.

117. Kim LS, Hilli L, Orlowski J, Kupperman JL, Baral M, Waters RF. Efficacy of probiotics and nutrients in functional gastrointestinal disorders: a preliminary clinical trial. Dig Dis Sci. 2006;51(12):2134-44.

118. Ohtsu T, Takagi A, Uemura N, Inoue K, Sekino H, Kawashima A, Uchida M, Koga Y. The Ameliorating Effect of Lactobacillus gasseri OLL2716 on Functional Dyspepsia in Helicobacter pylori-Uninfected Individuals: A Randomized Controlled Study. Digestion. 2017;96:92-102.

119. Xu D, Gao J, Gillilland M, Wu X, Song I, Kao JY, Owyang C. Rifaximin alters intestinal bacteria and prevents stress-induced gut inflammation and visceral hyperalgesia in rats. Gastroenterology. 2014;146(2):484-96.

120. Tan VPY, Liu KSH, Lam FYF, Hung IF, Yuen MF, Leung WK. Randomised clinical trial: rifaximin versus placebo for the treatment of functional dyspepsia. Aliment Pharmacol Ther. 2017;45:767-76.

121. Cao J, Ren X, Zhu G. Sa1 116 Significance of Double Steps Reattribution Integrative Model for Patients With Functional Dyspepsia (FD). Gastroenterology. 2013;5(1):S205-6.

122. Haag S, Senf W, Tagay S, Langkafel M, Braun-Lang U, Pietsch A, Heuft G, Talley NJ, Holtmann G. Is there a benefit from intensified medical and psychological interventions in patients with functional dyspepsia not responding to conventional therapy? Aliment Pharmacol Ther. 2007;25:973-86.

123. Jiang HMn, Jiang YB, Zhang SH. The effect of psychotherapy intervention on pharmacotherapy of patients with functional dyspepsia. Pharmaceutical Care and Research. 2008;8(1):52-4.
124. Orive M, Barrio I, Orive VM, Matellanes B, Padierna JA, Cabriada J, Orive A, Escobar A, Quintana JM. A randomized controlled trial of a 10 week group psychotherapeutic treatment added to standard medical treatment in patients with functional dyspepsia. J Psychosom Res. 2015;78:563-8.

125. Parkman HP, Hasler WL, Fisher RS. American Gastroenterological Association medical position statement: Diagnosis and treatment of gastroparesis. Gastroenterology. 2004;127:1589-91.

126. Camilleri M, Parkman HP, Shafi MA, Abell TL, Gerson L; American College of Gastroenterology. Clinical guideline: management of gastroparesis. Am J Gastroenterol. 2013;108:18-37.

127. Jung HK, Choung RS, Locke GR, Schleck CD, Zinsmeister AR, Szarka LA, Mullan B, Talley NJ. The incidence, prevalence, and outcomes of patients with gastroparesis in Olmsted County, Minnesota, from 1996 to 2006. Gastroenterology. 2009;136:1225-33.

128. Rey E, Choung RS, Schleck CD, Zinsmeister AR, Talley NJ, Locke GR. Prevalence of hidden gastroparesis in the community: the gastroparesis 'iceberg'. J Neurogastroenterol Motil. 2012;18:34-42.

129. Soykan I, Sivri B, Sarosiek I, Kiernan B, McCallum RW. Demography, clinical characteristics, psychological and abuse profiles, treatment, and long-term follow-up of patients with gastroparesis. Dig Dis Sci. 1998;43:2398-404.

130. Camilleri M, Chedid V, Ford AC, Haruma K, Horowitz M, Jones KL, Low PA, Park SY, Parkman HP, Stanghellini V. Gastroparesis. Nature Reviews Disease Primers. 2018;4(1):41.

131. Vassallo M, Camilleri M, Caron BL, Low PA. Gastrointestinal motor dysfunction in acquired selective cholinergic dysautonomia associated with infectious mononucleosis. Gastroenterology. 1991;100:252-8.

132. Bekkelund M, Sangnes DA, Gunnar Hatlebakk J, Aabakken L. Pathophysiology of idiopathic gastroparesis and implications for therapy. Scand J Gastroenterol. 2019;54:8-17.

133. Grover M, Farrugia G, Lurken MS, Bernard CE, Faussone-Pellegrini MS, Smyrk TC, Parkman HP, Abell TL, Snape WJ, Hasler WL, Ünalp-Arida A, Nguyen L, Koch KL, Calles J, Lee L, Tonascia J, Hamilton FA, Pasricha PJ; NIDDK Gastroparesis Clinical Research Consortium. Cellular changes in diabetic and idiopathic gastroparesis. Gastroenterology. 2011;140:1575-85.

134. Battaglia E, Bassotti G, Bellone G, Dughera L, Serra AM, Chiusa L, Repici A, Mioli P, Emanuelli G. Loss of interstitial cells of Cajal network in severe idiopathic gastroparesis. World J Gastroenterol. 2006;12:6172-7.

135. Bernard CE, Gibbons SJ, Mann IS, Froschauer L, Parkman HP, Harbison S, Abell TL, Snape WJ, Hasler WL, McCallum RW, Sarosiek I, Nguyen LA, Koch KL, Tonascia J, Hamilton FA, Kendrick ML, Shen KR, Pasricha PJ, Farrugia G; NIDDK Gastroparesis Clinical Research Consortium (GpCRC). Association of low numbers of CD206-positive cells with loss of ICC in the gastric body of patients with diabetic gastroparesis. Neurogastroenterol Motil. 2014;26:1275-84.

136. Grover M, Bernard CE, Pasricha PJ, Parkman HP, Gibbons SJ, Tonascia J, Koch KL, McCallum RW, Sarosiek I, Hasler WL, Nguyen LAB, Abell TL, Snape WJ, Kendrick ML, Kellogg TA, McKenzie TJ, Hamilton FA, Farrugia G; NIDDK Gastroparesis Clinical Research Consortium (GpCRC). Diabetic and idiopathic gastroparesis is associated with loss of CD206-positive macrophages in the gastric antrum. Neurogastroenterol Motil. 2017;29(6):10. 
137. Sharma A, Parkman HP, Thomas R. Myenteric Ganglionitis in Gastroparesis. Gastroenterology. 2011;140:S-71.

138. Soota K, Kedar A, Nikitina Y, Arendale E, Vedanarayanan V, Abell TL. Immunomodulation for treatment of drug and device refractory gastroparesis. Results Immunol. 2016;6:11-4.

139. Cogliandro RF, Rizzoli G, Bellacosa L, De Giorgio R, Cremon C, Barbara G, Stanghellini V. Is gastroparesis a gastric disease? Neurogastroenterol Motil. 2019;31:e13562.

140. Talley NJ, Verlinden M, Jones M. Can symptoms discriminate among those with delayed or normal gastric emptying in dysmotility-like dyspepsia? Am J Gastroenterol. 2001;96:1422-8.

141. Karamanolis G, Caenepeel P, Arts J, Tack J. Determinants of symptom pattern in idiopathic severely delayed gastric emptying: gastric emptying rate or proximal stomach dysfunction? Gut. 2007;56:29-36.

142. Kumar A, Attaluri A, Hashmi S, Schulze KS, Rao SS. Visceral hypersensitivity and impaired accommodation in refractory diabetic gastroparesis. Neurogastroenterol Motil. 2008;20:635-42.

143. Frokjaer JB, Andersen LW, Brock C, Simrén M, Ljungberg M, Søfteland E, Dimcevski G, Yavarian Y, Gregersen H, Drewes AM. Altered brain microstructure assessed by diffusion tensor imaging in patients with diabetes and gastrointestinal symptoms. Diabetes Care. 2013;36:662-8.

144. Snodgrass P, Sandoval H, Calhoun VD, Ramos-Duran L, Song G, Sun Y, Alvarado B, Bashashati M, Sarosiek I, McCallum RW. Central Nervous System Mechanisms of Nausea in Gastroparesis: An fMRI-Based Case-Control Study. Dig Dis Sci. 2020;65:551-6.

145. Parkman HP, Yates K, Hasler WL, Nguyen L, Pasricha PJ, Snape WJ, Farrugia G, Koch KL, Calles J, Abell TL, McCallum RW, Lee L, Unalp-Arida A, Tonascia J, Hamilton F; National Institute of Diabetes and Digestive and Kidney Diseases Gastroparesis Clinical Research Consortium. Similarities and differences between diabetic and idiopathic gastroparesis. Clin Gastroenterol Hepatol. 2011;9:1056-64.

146. Choung RS, Locke GR, Schleck CD, Zinsmeister AR, Melton LJ, Talley NJ. Risk of gastroparesis in subjects with type 1 and 2 diabetes in the general population. Am J Gastroenterol. 2012;107:82-8.

147. Browning KN, Travagli RA. Central nervous system control of gastrointestinal motility and secretion and modulation of gastrointestinal functions. Compr Physiol. 2014;4:1339-68.

148. Du P, Grady GO, Paskaranandavadivel N, Tang SJ, Abell T, Cheng LK. High-resolution Mapping of Hyperglycemia-induced Gastric Slow Wave Dysrhythmias. J Neurogastroenterol Motil. 2019;25:276-85.

149. Bharucha AE, Camilleri M, Forstrom LA, Zinsmeister AR. Relationship between clinical features and gastric emptying disturbances in diabetes mellitus. Clin Endocrinol. 2009;70:415-20.

150. Halland M, Bharucha AE. Relationship Between Control of Glycemia and Gastric Emptying Disturbances in Diabetes Mellitus. Clin Gastroenterol Hepatol. 2016;14:929-36.

151. Van Rijn S, Roebroek YGM, Conchillo JM, Bouvy ND, Masclee AA. Effect of Vagus Nerve Injury on the Outcome of Antireflux Surgery: An Extensive Literature Review. Dig Surg. 2016;33:230-9.

152. Desprez C, Melchior C, Wuestenberghs F, Huet E, Zalar A, Jacques J, Leroi AM, Gourcerol G. Pyloric distensibility measurement after gastric surgery: Which surgeries are associated with pylorospasm? Neurogastroenterol Motil. 2020;32:e13790.

153. Frantzides CT, Carlson MA, Zografakis JG, Moore RE, Zeni T, Madan AK. Postoperative gastrointestinal complaints after laparoscopic Nissen fundoplication. JSLS. 2006;10:39-42.
154. Parkman HP, Hallinan EK, Hasler WL, Farrugia G, Koch KL, Calles J, Snape WJ, Abell TL, Sarosiek I, McCallum RW, Nguyen L, Pasricha PJ, Clarke J, Miriel L, Lee L, Tonascia J, Hamilton F; NIDDK Gastroparesis Clinical Research Consortium (GpCRC). Nausea and vomiting in gastroparesis: similarities and differences in idiopathic and diabetic gastroparesis. Neurogastroenterology \& Motility. 2016;28:1902-14.

155. Hasler WL, Wilson LA, Parkman HP, Nguyen L, Abell TL, Koch KL, Pasricha PJ, Snape WJ, Farrugia G, Lee L, Tonascia J, Unalp-Arida A, Hamilton F; NIDDK Gastroparesis Clinical Research Consortium (GpCRC). Bloating in gastroparesis: severity, impact, and associated factors. Am J Gastroenterol. 2011;106:1492-502.

156. Camilleri M, Lembo A, Katzka DA. Opioids in Gastroenterology: Treating Adverse Effects and Creating Therapeutic Benefits. Clin Gastroenterol Hepatol. 2017;15:1338-49.

157. Mittal RK, Frank EB, Lange RC, McCallum RW. Effects of morphine and naloxone on esophageal motility and gastric emptying in man. Digestive Diseases and Sciences. 1986;31:936-42.

158. Jeong ID, Camilleri M, Shin A, Iturrino J, Boldingh A, Busciglio I, Burton D, Ryks M, Rhoten D, Zinsmeister AR. A Randomized, Placebo-Controlled Trial Comparing the Effects of Tapentadol and Oxycodone on Gastrointestinal and Colonic Transit in Healthy Humans. Aliment Pharmacol Ther. 2012;35(9):1088-96.

159. Maurer AH, Krevsky B, Knight LC, Brown K. Opioid and opioid-like drug effects on whole-gut transit measured by scintigraphy. J Nucl Med. 1996;37:818-22.

160. Salehi M, Aulinger BA, D’Alessio DA. Targeting beta-cell mass in type 2 diabetes: promise and limitations of new drugs based on incretins. Endocr Rev. 2008;29:367-79.

161. Vella A, Bock G, Giesler PD, Burton DB, Serra DB, Saylan ML, Dunning BE, Foley JE, Rizza RA, Camilleri M. Effects of Dipeptidyl Peptidase-4 Inhibition on Gastrointestinal Function, Meal Appearance, and Glucose Metabolism in Type 2 Diabetes. Diabetes. 2007;56:1475-80.

162. Thorn AR. Not just another case of nausea and vomiting: a review of postinfectious gastroparesis. J Am Acad Nurse Pract. 2010;22:125-33.

163. Oh JJ, Kim CH. Gastroparesis After a Presumed Viral Illness: Clinical and Laboratory Features and Natural History. Mayo Clinic Proceedings. 1990;65:636-42.

164. Naftali T, Yishai R, Zangen T, Levine A. Post-infectious gastroparesis: Clinical and electerogastrographic aspects. Journal of Gastroenterology and Hepatology. 2007;22:1423-8.

165. Manetti M, Milia AF, Benelli G, Messerini L, Matucci-Cerinic M, Ibba-Manneschi L. The gastric wall in systemic sclerosis patients: a morphological study. Ital J Anat Embryol. 2010;115:115-21.

166. Soykan I, Sarosiek I, Sivri B, Kiernan B, McCallum RW. Demographic, clinical characteristics, psychologic profiles, treatment, long term follow up of patients with gastroparesis. Southern Medical Journal. 1998;91:S37.

167. Quigley EMM. Other forms of gastroparesis: postsurgical, Parkinson, other neurologic diseases, connective tissue disorders. Gastroenterol Clin North Am. 2015;44:69-81.

168. Choi SW, Kang SH, Kwon OS, Park HW, Lee S, Koo BS, Nam GB. A Case of Severe Gastroparesis: Indigestion and Weight Loss after Catheter Ablation of Atrial Fibrillation. Pacing and Clinical Electrophysiology. 2012;35(3):e59-61.

169. Youle MS, Read NW. Effect of painless rectal distension on gastrointestinal transit of solid meal. Digestive Diseases and Sciences. 1984;29:902-6. 
170. Tjeerdsma HC, Smout AJP, Akkermans LMA. Voluntary suppression of defecation delays gastric emptying. Digestive Diseases and Sciences. 1993;38:832-6.

171. Jeong ID, Camilleri M, Shin A, Iturrino J, Boldingh A, Busciglio I, Burton D, Ryks M, Rhoten D, Zinsmeister AR. A randomised, placebo-controlled trial comparing the effects of tapentadol and oxycodone on gastrointestinal and colonic transit in healthy humans. Aliment Pharmacol Ther. 2012;35:1088-96.

172. Palmer SE, McLean RM, Ellis PM, Harrison-Woolrych M. Life-threatening clozapine-induced gastrointestinal hypomotility: an analysis of 102 cases. J Clin Psychiatry. 2008;69:759-68.

173. Szarka LA, Camilleri M. Evaluation of Patients with Suspected Gastroparesis. Gastrointest Endosc Clin N Am. 2019;29:39-54.

174. Coleski R, Baker JR, Hasler WL. Endoscopic Gastric Food Retention in Relation to Scintigraphic Gastric Emptying Delays and Clinical Factors. Digestive Diseases and Sciences. 2016;61:2593-601.

175. Wilson P, Anselmino M, Hinder RA, Quigley EM. Ambulatory antroduodenal manometry in man: Development and validation. Gastroenterology. 1992;103:1402.

176. Tougas G, Eaker EY, Abell TL, Abrahamsson H, Boivin M, Chen J, Hocking MP, Quigley EM, Koch KL, Tokayer AZ, Stanghellini V, Chen Y, Huizinga JD, Rydén J, Bourgeois I, McCallum RW. Assessment of gastric emptying using a low fat meal: establishment of international control values. The American Journal of Gastroenterology. 2000;95:1456-62.

177. Camilleri M, Iturrino J, Bharucha AE, Burton D, Shin A, Jeong ID, Zinsmeister AR. Performance characteristics of scintigraphic measurement of gastric emptying of solids in healthy participants. Neurogastroenterology \& Motility. 2012;24:1076-e562.

178. Usai-Satta P, Bellini M, Morelli O, Geri F, Lai M, Bassotti G. Gastroparesis: New insights into an old disease. World J Gastroenterol. 2020;26:2333-48.

179. Grover M, Farrugia G, Stanghellini V. Gastroparesis: a turning point in understanding and treatment. Gut. 2019;68:2238-50.

180. Szarka LA, Camilleri M, Vella A, Burton D, Baxter K, Simonson J, Zinsmeister AR. A Stable Isotope Breath Test With a Standard Meal for Abnormal Gastric Emptying of Solids in the Clinic and in Research. Clinical Gastroenterology and Hepatology. 2008;6:635-43.

181. Ghoos YF, Maes BD, Geypens BJ, Mys G, Hiele MI, Rutgeerts PJ, Vantrappen G. Measurement of gastric emptying rate of solids by means of a carbon-labeled octanoic acid breath test. Gastroenterology. 1993;104:1640-7.

182. Casteele M, Luypaerts A, Geypens B, Fevery J, Ghoos Y, Nevens F. Oxidative breakdown of octanoic acid is maintained in patients with cirrhosis despite advanced disease. Neurogastroenterology and Motility. 2003;15:113-20.

183. Maqbool S, Parkman HP, Friedenberg FK. Wireless Capsule Motility: Comparison of the SmartPill GI Monitoring System with Scintigraphy for Measuring Whole Gut Transit. Digestive Diseases and Sciences. 2009;54:2167-74.

184. Kuo B, McCallum RW, Koch KL, Sitrin MD, Wo JM, Chey WD, Hasler WL, Lackner JM, Katz LA, Semler JR, Wilding GE, Parkman HP. Comparison of gastric emptying of a nondigestible capsule to a radio-labelled meal in healthy and gastroparetic subjects. Alimentary Pharmacology \& Therapeutics. 2007;27:186-96.
185. Fidler J, Bharucha AE, Camilleri M, Camp J, Burton D, Grimm R, Riederer SJ, Robb RA, Zinsmeister AR. Application of magnetic resonance imaging to measure fasting and postprandial volumes in humans. Neurogastroenterol Motil. 2009;21:42-51.

186. Bolondi L, Bortolotti M, Santi V, Calletti T, Gaiani S, Labò G. Measurement of gastric emptying time by real-time ultrasonography. Gastroenterology. 1985;89:752-9.

187. Camilleri M. Functional Dyspepsia and Gastroparesis. Dig Dis. 2016;34:491-9.

188. Grover M, Farrugia G, Stanghellini V. Gastroparesis: a turning point in understanding and treatment. Gut. 2019;68:2238-50.

189. Homko CJ, Duffy F, Friedenberg FK, Boden G, Parkman HP. Effect of dietary fat and food consistency on gastroparesis symptoms in patients with gastroparesis. Neurogastroenterol Motil. 2015;27:501-8.

190. Parkman HP, Yates KP, Hasler WL, Nguyan L, Pasricha PJ, Snape WJ, Farrugia G, Calles J, Koch KL, Abell TL, McCallum RW, Petito D, Parrish CR, Duffy F, Lee L, Unalp-Arida A, Tonascia J, Hamilton F; NIDDK Gastroparesis Clinical Research Consortium. Dietary intake and nutritional deficiencies in patients with diabetic or idiopathic gastroparesis. Gastroenterology. 2011;141:486-98.

191. Bharucha AE, Kudva Y, Basu A, Camilleri M, Low PA, Vella A, Zinsmeister AR. Relationship between glycemic control and gastric emptying in poorly controlled type 2 diabetes. Clin Gastroenterol Hepatol. 2015;13:466-76.

192. Lata PF, Pigarelli DLW. Chronic metoclopramide therapy for diabetic gastroparesis. Ann Pharmacother. 2003;37:122-6.

193. Cavero Redondo I, Álvarez Bueno C, Pozuelo Carrascosa DP, Díez Fernández A, Notario Pacheco B. Risk of extrapyramidal side effects comparing continuous vs. bolus intravenous metoclopramide administration: a systematic review and meta-analysis of randomised controlled trials. J Clin Nurs. 2015;24:3638-46.

194. Rao AS, Camilleri M. Review article: metoclopramide and tardive dyskinesia. Alimentary Pharmacology \& Therapeutics. 2010;31:11-9.

195. Sturm A, Holtmann G, Goebell H, Gerken G. Prokinetics in patients with gastroparesis: a systematic analysis. Digestion. 1999;60:422-7.

196. Maganti K, Onyemere K, Jones MP. Oral erythromycin and symptomatic relief of gastroparesis: a systematic review. Am J Gastroenterol. 2003;98:259-63.

197. Richards RD, Davenport K, McCallum RW. The treatment of idiopathic and diabetic gastroparesis with acute intravenous and chronic oral erythromycin. Am J Gastroenterol. 1993;88:203-7.

198. Navas CM, Patel NK, Lacy BE. Symptomatic Management of Gastroparesis. Gastrointest Endosc Clin N Am. 2019;29:55-70.

199. Camilleri M, Beyens G, Kerstens R, Robinson P, Vandeplassche L. Safety assessment of prucalopride in elderly patients with constipation: a double-blind, placebo-controlled study. Neurogastroenterol Motil. 2009;21:1256-e117.

200. Tack J, Camilleri M, Chang L, Chey WD, Galligan JJ, Lacy BE, Müller-Lissner S, Quigley EM, Schuurkes J, De Maeyer JH, Stanghellini V. Systematic review: cardiovascular safety profile of 5-HT(4) agonists developed for gastrointestinal disorders. Aliment Pharmacol Ther. 2012;35:745-67. 
201. Carbone F, Van den Houte K, Clevers E, Andrews CN, Papathanasopoulos A, Holvoet L, Van Oudenhove L, Caenepeel P, Arts J, Vanuytsel T, Tack J. Prucalopride in Gastroparesis: A Randomized Placebo-Controlled Crossover Study. Am J Gastroenterol. 2019;114:1265-74.

202. Kuo B, Barnes CN, Nguyen DD, Shaywitz D, Grimaldi M, Renzulli C, Canafax D, Parkman HP. Velusetrag accelerates gastric emptying in subjects with gastroparesis: a multicentre, double-blind, randomised, placebo-controlled, phase 2 study. Aliment Pharmacol Ther. 2021;53:1090-7.

203. Lembo A, Camilleri M, McCallum R, Sastre R, Breton C, Spence S, White J, Currie M, Gottesdiener K, Stoner E; RM-131-004 Trial Group. Relamorelin Reduces Vomiting Frequency and Severity and Accelerates Gastric Emptying in Adults With Diabetic Gastroparesis. Gastroenterology. 2016;151:87-96.

204. Camilleri M, McCallum RW, Tack J, Spence SC, Gottesdiener K, Fiedorek FT. Efficacy and Safety of Relamorelin in Diabetics With Symptoms of Gastroparesis: A Randomized, Placebo-Controlled Study. Gastroenterology. 2017;153:1240-50.

205. Liu T, Ji R-R. New insights into the mechanisms of itch: are pain and itch controlled by distinct mechanisms? Pflugers Arch. 2013;465:1671-85.

206. Jacob D, Busciglio I, Burton D, Halawi H, Oduyebo I, Rhoten D, Ryks M, Harmsen WS, Camilleri M. Effects of NK1 receptors on gastric motor functions and satiation in healthy humans: results from a controlled trial with the NK1 antagonist aprepitant. Am J Physiol Gastrointest Liver Physiol. 2017;313(5):G505-G510.

207. Jehangir A, Parkman HP. Cannabinoid Use in Patients With Gastroparesis and Related Disorders: Prevalence and Benefit. Am J Gastroenterol. 2019;114:945-53.

208. Parkman HP, Van Natta ML, Abell TL, McCallum RW, Sarosiek I, Nguyen L, Snape WJ, Koch KL, Hasler WL, Farrugia G, Lee L, Unalp-Arida A, Tonascia J, Hamilton F, Pasricha PJ. Effect of nortriptyline on symptoms of idiopathic gastroparesis: the NORIG randomized clinical trial. JAMA. 2013;310:2640-9.

209. Malamood M, Roberts A, Kataria R, Parkman HP, Schey R. Mirtazapine for symptom control in refractory gastroparesis. Drug Des Devel Ther. 2017;11:1035-41.

210. Melga P, Mansi C, Ciuchi E, Giusti R, Sciaba L, Prando R. Chronic administration of levosulpiride and glycemic control in IDDM patients with gastroparesis. Diabetes Care. 1997;20:55-8.

211. Mansi C, Borro P, Giacomini M, Biagini R, Mele MR, Pandolfo N, Savarino V. Comparative effects of levosulpiride and cisapride on gastric emptying and symptoms in patients with functional dyspepsia and gastroparesis. Aliment Pharmacol Ther. 2000;14:561-9.

212. Kim BJ, Kuo B. Gastroparesis and Functional Dyspepsia: A Blurring Distinction of Pathophysiology and Treatment. J Neurogastroenterol Motil. 2019;25:27-35.

213. Levinthal DJ, Bielefeldt K. Systematic review and meta-analysis: Gastric electrical stimulation for gastroparesis. Auton Neurosci. 2017;202:45-55.
214. Abell TL, Yamada G, McCallum RW, Van Natta ML, Tonascia J, Parkman HP, Koch KL, Sarosiek I, Farrugia G, Grover M, Hasler W, Nguyen L, Snape W, Kuo B, Shulman R, Hamilton FA, Pasricha PJ. Effectiveness of gastric electrical stimulation in gastroparesis: Results from a large prospectively collected database of national gastroparesis registries. Neurogastroenterol Motil. 2019;31(12):e13714.

215. Ducrotte P, Coffin B, Bonaz B, Fontaine S, Bruley Des Varannes S, Zerbib F, Caiazzo R, Grimaud JC, Mion F, Hadjadj S, Valensi PE, Vuitton L, Charpentier G, Ropert A, Altwegg R, Pouderoux P, Dorval E, Dapoigny M, Duboc H, Benhamou PY, Schmidt A, Donnadieu N, Gourcerol G, Guerci B; ENTERRA Research Group. Gastric Electrical Stimulation Reduces Refractory Vomiting in a Randomized Crossover Trial. Gastroenterology. 2020;158:506-14.

216. Malik Z, Sankineni A, Parkman HP. Assessing pyloric sphincter pathophysiology using EndoFLIP in patients with gastroparesis. Neurogastroenterol Motil. 2015;27:524-31.

217. Gourcerol G, Tissier F, Melchior C, Touchais JY, Huet E, Prevost G, Leroi AM, Ducrotte P. Impaired fasting pyloric compliance in gastroparesis and the therapeutic response to pyloric dilatation. Aliment Pharmacol Ther. 2015;41:360-7.

218. Arts J, Van Gool S, Caenepeel P, Verbeke K, Janssens J, Tack $\mathrm{J}$. Influence of intrapyloric botulinum toxin injection on gastric emptying and meal-related symptoms in gastroparesis patients. Aliment Pharmacol Ther. 2006;24:661-7.

219. Bromer MQ, Friedenberg F, Miller LS, Fisher RS, Swartz K, Parkman HP. Endoscopic pyloric injection of botulinum toxin A for the treatment of refractory gastroparesis. Gastrointest Endosc. 2005;61(7):833-9.

220. Friedenberg FK, Palit A, Parkman HP, Hanlon A, Nelson DB. Botulinum toxin A for the treatment of delayed gastric emptying. Am J Gastroenterol. 2008;103:416-23.

221. Arts J, Holvoet L, Caenepeel P. Clinical trial: a randomized-controlled crossover study of intrapyloric injection of botulinum toxin in gastroparesis. Aliment Pharmacol Ther. 2007;26(9):1251-8.

222. Khashab MA, Stein E, Clarke JO, Saxena P, Kumbhari V, Chander Roland B, Kalloo AN, Stavropoulos S, Pasricha P, Inoue H. Gastric peroral endoscopic myotomy for refractory gastroparesis: first human endoscopic pyloromyotomy (with video). Gastrointest Endosc. 2013;78:764-8.

223. Mekaroonkamol P, Dacha S, Patel V, Li B, Luo H, Shen S, Chen H, Cai Q. Outcomes of Per Oral Endoscopic Pyloromyotomy in the United States. Gastrointest Endosc Clin N Am. 2019;29:151-60.

224. Aghaie Meybodi M, Qumseya BJ, Shakoor D, Lobner K, Vosoughi K, Ichkhanian Y, Khashab MA. Efficacy and feasibility of G-POEM in management of patients with refractory gastroparesis: a systematic review and meta-analysis. Endosc Int Open. 2019;7:E322-9.

225. Mohan BP, Chandan S, Jha LK, Khan SR, Kotagiri R, Kassab LL, Ravikumar NPG, Bhogal N, Chandan OC, Bhat I, Hewlett AT, Jacques J, Ponnada S, Asokkumar R, Adler DG. Clinical efficacy of gastric per-oral endoscopic myotomy (G-POEM) in the treatment of refractory gastroparesis and predictors of outcomes: a systematic review and meta-analysis using surgical pyloroplasty as a comparator group. Surg Endosc. 2020;34:3352-67. 